\title{
Valoración no monetaria de unidades de paisaje en la zona maya de Quintana Roo, México
}

\section{Non-monetary valuation of landscape units in the mayan zone of Quintana Roo, Mexico}

\author{
Karla Diana Infante-Ramírez* \\ Ana Minerva Arce-Ibarra* \\ EduARdo Bello-BaltaZAR**
}

\begin{abstract}
Our study was aimed at assessing the local value of landscapes from ejidos in the Mayan Zone of Quintana Roo by means of the damage schedule approach. The valuation was based upon the opinions of community groups as well as from the expertise of several scholars with previous research at the study area. Based on the opinion of the interviewees, our results show that mature rainforest was the most valued landscape unit, whereas wetlands were the least valued. These results were directly related to the number of activities undertaken at these landscape units which satisfy the subsistence needs of the Maya.
\end{abstract}

Key words: value, ecological economics, maya, rainforest, landscape unit.

\section{Resumen}

El objetivo de esta investigación es conocer el valor local del paisaje en ejidos de la zona maya de Quintana Roo. Se utilizó un método de valoración nomonetaria, el cual se basa tanto en la opinión de grupos comunitarios como de expertos académicos. Además, se apoyó en la observación participante, entrevistas a fondo y talleres comunitarios. Los resultados muestran que, en opinión de los entrevistados, el monte alto fue la unidad de paisaje más valorada y la sabana la menos valorada. Lo anterior coincide con el potencial que tienen las unidades para satisfacer las necesidades de subsistencia de las comunidades mayas estudiadas.

Palabras clave: valor, economía ecológica, mayas, selva, unidad de paisaje.

* El Colegio de la Frontera Sur, unidad Chetumal. Correos-e: kinfante@ecosur.mx y aarce@ ecosur.mx

** El Colegio de la Frontera Sur, unidad San Cristóbal de Las Casas. Correo-e: ebello@ecosur.mx 


\section{Introducción}

El paisaje se identifica como la síntesis de los sistemas ecológicos y culturales que lo constituyen, es decir, como la expresión visual de los fenómenos relacionados en la interacción sociedad-naturaleza (López-Barajas y Cervantes-Borja, 2002). El mosaico actual que constituyen los paisajes es producto innegable de la historia; incluye huellas de los efectos culturales que surgen a diferentes escalas espaciales y temporales (Nassauer, 1997). Los componentes espaciales están condicionados por una combinación de factores biofísicos y socioeconómicos que confluyen en el territorio; algunos efectos se identifican claramente en paisajes que han sido modificados y formados por razones productivas como los paisajes rurales (Lafortezza et al., 2008). Son una construcción de generaciones sucesivas de experimentación y modificación humana, de negociación con los elementos materiales y los procesos biofísicos que definen sus rasgos topográficos, hidrográficos y geomorfológicos; además, también son consecuencia de prácticas e imperativos ideológicos específicos (Buxó, 2006).

En las economías de subsistencia ligadas al medio natural, es decir, donde se toma directamente de la naturaleza todo lo necesario para vivir, se aprecia de manera evidente la dependencia humana de los ecosistemas (Gómez-Baggethum y De Groot, 2007). De manera particular, en muchos sistemas de conocimiento indígena sobre el manejo de los recursos naturales se incluyen también las dimensiones religiosas o espirituales (Berkes, 1999). En este sentido, las percepciones locales sobre la naturaleza y en particular sobre los ecosistemas y las unidades de paisaje pueden estar expresadas a través de leyendas, creencias, tradiciones y mitos, los cuales nos ayudan a establecer los límites entre las prácticas construidas en la interrelación sociedad-naturaleza (Lazos y Paré, 2000). Para los pueblos indígenas, la tierra, y en general, la naturaleza, tienen una cualidad sagrada que está casi ausente en el pensamiento occidental. La tierra es venerada y respetada, y su inalienabilidad se refleja en casi todas las cosmovisiones indígenas; las cuales no consideran a la tierra meramente como un recurso económico; así que bajo sus cosmovisiones, la naturaleza es la fuente primaria de vida que nutre, sostiene y enseña (Toledo, 2001).

El paisaje de la zona maya de la península de Yucatán se ha definido como un mosaico de vegetación en diferentes estados de sucesión y con distinta composición, que son el resultado de estrategias campesinas diversificadas (García-Frapolli et al., 2008). Estas estrategias de uso múltiple de los recursos naturales locales les permiten a las comunidades mantener una economía dual basada en la producción para la autosubsistencia, con porciones extraordinarias de esa producción dirigidas a los mercados (Toledo et al., 2008). Por un lado, producen y venden algunos 
bienes y servicios en el mercado como miel de abeja, carbón vegetal, artesanías y servicios ambientales (ecoturismo) y, a su vez, adquieren una serie de productos en el mercado tales como maíz, café, vestido, utensilios de trabajo y hasta coches. Por otro lado, producen, a manera de autoconsumo, maíz (Zea mays), frijol (Phaseolus spp), calabaza (Cucurbita spp), chiles (Capsicum spp) y otras frutas y verduras, además de recolectar agua subterránea, leña, plantas medicinales y madera para la construcción de casas, en la mayoría de los casos bajo un esquema de bienes comunales (García-Frapolli et al., 2008).

La parte centro-sur de la península de Yucatán se ha identificado como una zona importante para la implementación de proyectos y programas de conservación de la selva maya, tanto en la esfera nacional como en la internacional; debido a que representa una de las zonas boscosas de mayor amplitud y diversidad de México, sin embargo, también se encuentran altos índices de pobreza (Faust, 2001; Porter-Bolland et al., 2007). Para esta y otras zonas se reconoce que actualmente la toma de decisiones se relaciona con la posibilidad de privilegiar las ganancias en el corto plazo (López-Hernández et al., 2012, Araújo-Santana et al., 2013).

En particular, Faust (2001) y Bello-Baltazar (2001) han destacado que en la zona maya de la península de Yucatán se han implementado proyectos gubernamentales que responden a una lógica de mercado que no ha considerado las características ecológicas y culturales de la región, trayendo como consecuencia fracasos importantes en el ámbito ambiental, cultural y económico. Ante estos desaciertos, se identifica una necesidad de buscar alternativas para la valoración de los bienes ambientales, que consideren los valores que la gente asocia a lugares o paisajes (Brown y Raymond, 2007), lo cual permitiría la inclusión de los diferentes actores desde un enfoque de abajo-arriba, para respaldar procesos de toma de decisiones más equitativos en la gestión de los recursos naturales.

En este sentido, la valoración local desempeña un papel importante en la planificación del uso y manejo de los recursos naturales. La valoración alternativa de los ecosistemas y del paisaje se ha vuelto parte esencial para llevar a cabo una utilización sostenible de los recursos naturales y mantener el bienestar humano (Grêt-Regamey et al., 2008). En general, si se toman en cuenta los valores locales, las políticas gubernamentales pueden adecuarse mejor a las necesidades públicas y, cuando los valores se relacionan con los indicadores científicos de las condiciones del ecosistema, se puede reconocer y actuar sobre las amenazas al medio ambiente (Patel et al., 1999; Vera-Méndez, 2008). 


\section{Valoración del ambiente}

Existen varias disciplinas desde las cuales se puede valorar el ambiente, entre ellas la economía ambiental y la economía de los recursos naturales, las cuales se derivan de la teoría económica neoclásica en el sentido de que ambas utilizan métodos de valoración de la naturaleza usando lenguajes monetarios y cuyas teorías se basan en la racionalidad económica (Fuente-Carrasco, 2008).

Entre sus métodos se encuentran el análisis costo-beneficio, el costo del viaje y el de valoración contingente (Kramer et al., 1996; Nahuelhual et al., 2006); este último se emplea especialmente para evaluar daños al ambiente, creando mercados hipotéticos, pues generalmente no se tiene una idea previa del costo del daño causado, por ejemplo, por un derrame de petróleo en un ecosistema terrestre o marino. La valoración contingente y sus resultados han sido usados comúnmente en juicios legales en donde se pide una compensación económica por un daño ambiental.

Por otro lado, existe el campo emergente de la economía ecológica, la cual se reconoce, entre otras, porque su teoría se basa en una racionalidad ambiental (considerando siempre los límites biofísicos) y sus métodos no se fundamentan en valoraciones monetarias (Granato et al., 2009). Además, es un campo reconocido como multi, inter o transdisciplinario, dado que usa el conocimiento en su teoría y en sus métodos de valoración de varias disciplinas como la física, la economía, la ecología, etc. FuenteCarrasco (2008) menciona que la economía ecológica (EE) sigue en formación y que actualmente cuenta con varias corrientes teóricas, entre las que destacan: a) la EE conservadora, que se identifica porque no desea abordar temas relacionados con conflictos sociales (lucha de clases) y en cambio se enfoca en abordar los aspectos económicos del mercado y los aspectos ecológicos del ambiente; b) la EE crítica, que reconoce que la racionalidad económica neoclásica genera insustentabilidad y que por ello explora otras alternativas a tal racionalidad. Además, aquí se aborda el tema del conflicto ecológico distributivo (distribución inequitativa de los beneficios de la economía neoclásica); y c) la EE heterodoxa o radical, que usa marcos teóricos para entender las causas estructurales de la aparente contradicción entre la sociedad y la naturaleza, y que, además del conocimiento científico, integra los saberes de las prácticas campesinas.

Este estudio utiliza un método de valoración llamado esquema de daños y pérdidas (damage schedule approach), el cual fue originalmente propuesto en revistas usadas en foros legales como una alternativa a los problemas de los métodos de valoración existentes y, en particular, del método de valoración contingente (Rutherford et al., 1998), el cual considera las escalas de importancia de un bien ambiental derivadas de la 
opinión social, reflejando así los valores que un grupo o una comunidad le otorga a los bienes ambientales y que por lo tanto deberían ser consideradas en su conservación y manejo.

Desde su primera propuesta, en 1998, y sus posteriores aplicaciones (Chuenpagdee, 1998; Chuenpagdee et al., 2002) este método ha tenido algunas variaciones, Arce-Ibarra (2007) le adiciona fotografías de los bienes ambientales que son objeto de evaluación. Después de una revisión de los fundamentos de la economía ambiental, la economía de los recursos naturales y de la economía ecológica, algunas universidades de países del hemisferio norte incluyen este método dentro de sus cursos de economía ecológica. Lo anterior por dos razones: primero, porque basa su valoración en unidades no monetarias y, segundo, porque al basarse en la opinión social tiene la flexibilidad de usar tanto el conocimiento científico como el de la gente local (de grupos sociales incluyendo colectivos, ejidos, etnias, entre otros). Además, como se verá más adelante, nuestro estudio le añade el criterio ecológico al método de esquema de daños y pérdidas, dado que usa la funcionalidad ecológica de un bien ambiental llamado unidad de paisaje.

Para valorar los recursos naturales, los economistas separan su valor en dos grupos: valor de uso y valor de no uso (Barbier, 1994; Chuenpagdee, 1998); los primeros pueden ser directos o indirectos, los más comunes son los de uso directo, por ejemplo, la extracción de madera para muebles; es decir, este valor se determina a partir de la utilización actual que se da al bien ambiental, mientras que el del uso indirecto beneficia a los individuos sin que en ocasiones se tenga conciencia de ello, y guarda relación con las funciones ecológicas que cumple el bien en cuestión, por ejemplo, el uso indirecto de la biosfera es un bien que nos asegura la condición de vida sobre la tierra sin que muchos tengan conciencia de ello. Aun así, el valor de uso indirecto no deja de ser un concepto funcionalista, el cual supone que el bien trabaja en función de nosotros, y es finalmente algo para nuestro uso.

Si se aplican las anteriores definiciones de valor al paisaje, los valores de no uso representan el valor intrínseco de la naturaleza y su asignación o reconocimiento tiene como propósito conservar la naturaleza viva o inerte independiente de cualquier utilidad, a su vez, este valor se divide en valor de existencia y en valor de opción, el primero, es el que otorgan algunas personas a la conservación del bien ambiental porque aun cuando no hacen ni harán uso de él, desean que exista. Mientras que el de opción se refiere al valor que se le da al área o al bien ambiental afectado por sus posibles usos futuros (por ejemplo, para uso de generaciones venideras). De todos estos valores, el más difícil de medir es el valor de existencia, por el hecho de que contempla los llamados beneficios pasivos 
(Barbier, 1994; Chuenpagdee, 1998). Esta clasificación nos permite sistematizar los valores que la gente otorga a los recursos naturales mediante su uso o no uso; como se puede apreciar, no todos los valores pueden tener un equivalente monetario, de ahí la relevancia de usar métodos de valoración no monetarios.

En la zona maya del centro de Quintana Roo las comunidades indígenas están organizadas en colectivos llamados ejidos; en los que se hace uso de una gama de recursos derivados de la selva entre los que se encuentran los policultivos como la milpa bajo el sistema de roza-tumba y quema, la extracción de maderas preciosas, duras y blandas, la extracción de resina (chicle) del zapote, el aprovechamiento de animales de monte (sereque, venado, jabalí, entre otros), así como de peces de los cuerpos de agua localizados en zonas inundables de la selva y en las sabanas (BelloBaltazar, 2001; Estrada-Lugo, 2005; Estrada-Lugo y Arce-Ibarra, 2011; Rojas-García 1999; Arce-Ibarra, 2007; Santos-Fita et al., 2012). El uso de los recursos de la selva se refleja en la transformación y reconocimiento de los elementos del paisaje local por la actividad de hombres, mujeres, jóvenes y nińos en los grupos locales de parentesco. Se trata de un proceso de transformación continuo que ha redundado a nivel de la comunidad en la conformación del territorio maya. Este proceso implica establecer fronteras, negociar espacios, atribuir identidades y recrear, a través de un ritual agrícola, mediaciones culturales (Estrada-Lugo, 2005).

Así también, Santiago-Cruz (2000), Bello-Baltazar (2001), MacarioMendoza (2003) y Estrada-Lugo (2005) mencionan que el paisaje en la zona maya puede representarse esquemáticamente mediante el uso actual del suelo como la base territorial que conforma el espacio social de la comunidad, el cual es reconocido y legitimado por los pobladores locales como propio a través del uso cotidiano, e identificado por las comunidades vecinas como tal. Estos autores también advierten que el reconocimiento de los elementos del paisaje se expresa en la designación local que dan a éstos, por ejemplo, el monte alto es nombrado noh k'aax, las áreas de vegetación secundaria reciben el nombre genérico de hubché, la sabana es chac ka'an, entre otros. El paisaje en la zona maya es entonces el resultado de la actividad productiva o extractiva de los mayas, resultando en paisajes altamente modificados, inmersos en una matriz de vegetación con diferentes grados de conservación.

El presente estudio se llevó a cabo en territorios indígenas mayas caracterizados por poseer una diversidad de ambientes, por lo que se consideró relevante ir más allá de una valoración de recursos naturales específicos o aislados, y concentrarse en unidades de paisaje que se constituyen como la unidad de análisis integral. De acuerdo con Mejía-Ávila (2007:10), estas unidades se definen como "una porción de la superficie 
terrestre con patrones de homogeneidad, conformada por un conjunto complejo de sistemas, producto de la actividad de las rocas, el agua, el aire, las plantas, los animales y el hombre, que por su fisonomía es reconocible y diferenciable de otras vecinas". Por lo tanto, la unidad de paisaje nos permite integrar elementos ecológicos, de organización social, económicos, entre otros que ayudan a comprender la relación sociedadnaturaleza.

Bajo estos preceptos, la investigación tuvo dos objetivos: el primero consistió en llevar a cabo una valoración no monetaria de los bienes ambientales del centro de Quintana Roo, la cual incluyó a un conjunto de unidades de paisaje de tres ejidos mayas y tuvo como insumo la opinión de un grupo comunitario por ejido, además de un equipo de investigadores que durante varios ańos han estudiado la zona; el segundo, tuvo como fin generar información como línea de base para el desarrollo del área seleccionada, partiendo del conocimiento local y su relación con el conocimiento científico para la toma de decisiones en el manejo del paisaje de la zona maya.

\section{2. Área de estudio. Localización, ambiente físico y población}

El área de estudio se localiza en el centro del estado de Quintana Roo en la región denominada zona maya entre las coordenadas 19²0'00" latitud N y 8805’00" longitud $\mathrm{O}$ dentro de la península de Yucatán en el caribe mexicano. Esta área es parte del Corredor Biológico Mesoamericano (СвмА) de iniciativa internacional en el municipio indígena de Felipe Carrillo Puerto (figura 1). La zona está formada por los descendientes de los mayas rebeldes que participaron durante el conflicto armado del siglo XIX, conocido como la Guerra de castas (Bello-Baltazar, 2001).

La vegetación del área, de acuerdo con la clasificación de Miranda y Hernández-Xolocotzi (1963), se compone principalmente de selva alta perennifolia, selva mediana y baja perennifolia, selva alta y mediana subperennifolia y de sabana. Esta vegetación crece en suelos someros y pedregosos en donde predominan las asociaciones de suelos rendzinas y litosoles (Miranda y Hernández-Xolocotzi, 1963). Aquí se encuentra una alta diversidad de fauna local incluyendo poblaciones residentes y migrantes de aves, mamíferos como el jaguar (Panthera onca), el venado (Odoicoileus virginianus) y el pecarí (Tayassu tajacu), así como reptiles, entre ellos los cocodrilos (Crocodilus spp.), además de otras especies (ArceIbarra y Charles, 2008).

El comportamiento anual de la precipitación en el área se compone de tres temporadas: la de secas (febrero a mayo), la de lluvias (junio a 


\section{Figura 1}

\section{Ubicación del área de estudio}

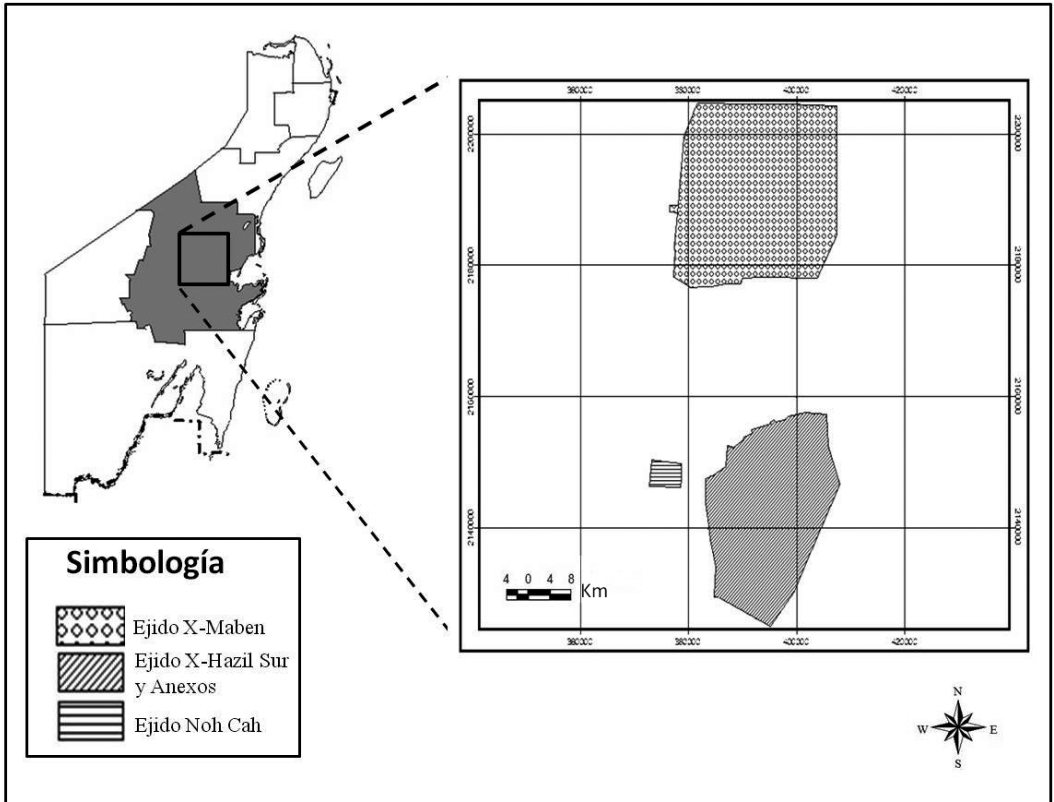

Fuente: elaboración propia.

octubre) y la de nortes (noviembre a febrero), aunque estas pueden sobreponerse (Pool et al., 2003; Arce-Ibarra y Charles, 2008).

El estudio se llevó a cabo en tres comunidades Noh Cah, Señor y XHazil sur que son los principales centros poblacionales de los ejidos Noh Cah, X-Maben y X-Hazil sur y anexos, respectivamente. El ejido Noh-Cah tiene una superficie de 2,600 hectáreas y cuenta con sólo una comunidad que lleva el mismo nombre del ejido, la cual, para 2005, tuvo una población total de 86 personas: 38 hombres y 48 mujeres (INEGI, 2006).

El ejido X-Maben cuenta con una superficie de 73,400 hectáreas y está conformado por seis localidades: Señor, San Antonio, Chanchen Comandante, San José, Noh Cancab y José María Pino Suárez, siendo Señor el principal centro poblacional del ejido y donde se encuentra la casa y el comisariado ejidales. En 2005 el número de habitantes de las localidades de X-Maben fue de: 2,872 en Señor; 40 en Noh Cancab; 59 en San Antonio; 13 Chanchen Comandante; ocho en San José, y 225 en José María Pino Suárez (Inegi, 2006). A su vez, el ejido X-Hazil sur y anexos cuenta con una superficie de 55,019 hectáreas y está conformado por tres centros de población: X-Hazil sur, Uh-May y Chancaj Veracruz (RAN, 1998). Según INEGi (2006) el número de habitantes por localidad 
de este ejido fue de 1,305 en X-Hazil sur, 425 en Uh May, y 356 en Chancaj, Veracruz.

\section{Métodos}

De acuerdo con el método de esquema de daños y pérdidas, el estudio se dividió en cuatro fases (Chuenpagdee, 1998), en la fase I se realizó un prediagnóstico en donde se delimitaron, seleccionaron y caracterizaron los bienes ambientales que serían objeto de la valoración. En nuestro caso, fueron las unidades de paisaje y para su delimitación se tomó en cuenta tanto el conocimiento local como el generado por científicos que durante varios años han investigado el área de estudio.

El proceso en su primera fase comprendió la selección de informantes clave para la elaboración de un mapa etnográfico para cada ejido en donde se mostrarían las diferentes áreas productivas en las que se realizan actividades específicas de cada una. Debido a que los participantes no se encontraban familiarizados con la terminología técnica unidad de paisaje, en lugar de ésta se utilizó el concepto espacios productivos del ejido. El propósito de este ejercicio fue, con base en el conocimiento local de los participantes, plasmar sobre una hoja grande de papel la visión gráfica y los conocimientos que ellos tienen de su entorno y de los recursos existentes en él (Bernard, 2006).

La delimitación de las unidades de paisaje se llevó a cabo identificando coincidencias entre las clasificaciones por uso de suelo, determinadas a partir del conocimiento científico y de la clasificación realizada por los informantes clave desde el conocimiento local en cada ejido, a través de mapas etnográficos. Al respecto, en las clasificaciones de uso de suelo realizadas por Santiago-Cruz (2000) y Macario-Mendoza (2003) en el ejido X-Hazil sur y anexos, uno de los elementos sobresalientes fue el tipo de vegetación y las características fisiográficas, en donde reportan 14 tipos de usos de suelo incluyendo dos para selva madura, tres para acahuales, dos para los cuerpos de agua, tres para la sabana y uno para potrero, campo citrícola y milpa. A su vez, Dalle et al. (2006) reportan para el ejido X-Maben una clasificación de siete usos de suelo: la milpa, los acahuales jóvenes, la vegetación secundaria, la selva baja caducifolia, la selva mediana perennifolia, los humedales y las zonas quemadas.

En contraste, para el ejido de Noh Cah, sólo se encontró la clasificación realizada en el (RAN, 1995) para el Programa de Certificación de Derechos Ejidales y Titulación de Solares Urbanos (Procede), en donde el ejido se divide en área parcelada, tierra de uso común, asentamientos humanos, infraestructura, cuerpos de agua y áreas especiales. Una vez delimitados los espacios productivos del ejido o las unidades de paisaje en cada uno se 
realizaron recorridos y se tomaron fotografías, mismas que fueron utilizadas para la elaboración de un cuadernillo que se usó durante la valoración no monetaria (Arce-Ibarra, 2007). Cabe resaltar que una vez delimitados los espacios a valorar, se realizaron entrevistas semiestructuradas a informantes clave para reforzar el reconocimiento de estos espacios ante la comunidad.

La fase II consistió en la elaboración del cuestionario de acuerdo con el método de comparación por pares (Rutherford et al., 1998), es decir, el cuestionario consistió en presentar cada una de sus preguntas con dos opciones (un par de unidades de paisaje) a elegir para cada participante. El número total de preguntas dependió del número de bienes ambientales (unidades de paisaje) seleccionados, el cual se basó en la siguiente fórmula (Dunn-Rankin et al., 2004):

$$
\mathrm{N}=\mathrm{n}(\mathrm{n}-1) / 2
$$

Donde $\mathrm{N}$ es el número total de preguntas (o número total de unidades presentadas en pares) y n es el número de unidades de paisajes seleccionadas para la valoración. Entonces, una vez que se selecciona el número de unidades a valorar (n), éste debe sustituirse en la fórmula (1) para obtener el número total de preguntas del cuestionario.

Al cuestionario se anexó el cuadernillo con las fotografías (un par en cada hoja) previamente tomadas en las unidades de paisaje de cada ejido y fue traducido al maya-yucateco, ya que la mayor parte de la población de los ejidos era maya-hablante. La metodología implicó presentar a cada participante dos escenarios de dańo a las unidades de paisaje que se deben tanto a causas naturales (huracanes) como a las actividades humanas (crecimiento de los poblados).

Así, cada pregunta del cuestionario se construyó en torno a un escenario de daño, es decir, se formularon cuestionamientos bajo la siguiente lógica: si nos imaginamos que las siguientes unidades de paisaje (se mostraban un par de fotos del cuadernillo) sufrieran algún daño (natural o inducido), ¿cuál de las dos cree usted que afectaría más a su comunidad? Este método tuvo como fin recabar el juicio de la gente al seleccionar un bien sobre el otro y darle mayor valor al objeto seleccionado (Vatn y Bromley, 1994; Rutherford et al., 1998).

De esta manera y, como se mencionó previamente, una vez que el participante seleccione una unidad sobre otra, está dando mayor valor a la seleccionada. Posteriormente, se cuenta el número de selecciones (o votos) para cada unidad y se ordenan del número mayor al menor, es decir, se obtiene una escala (de mayor a menor) de las preferencias individuales sobre las unidades de paisaje, las cuales reflejan la importancia o valor que tiene para cada participante. 
La fase III consistió en la aplicación del cuestionario utilizando un muestreo por cuota para obtener, al menos, 20 personas por grupo de interés que se dedicaran a determinadas actividades productivas o a trabajos similares (Chuenpagdee et al., 2002). Seleccionamos a tres grupos comunitarios de acuerdo con su estatus social y político: ejidatarios (con derechos de propiedad sobre la tierra), no ejidatarios (sin derechos de propiedad sobre la tierra), mujeres (ama de casa, con poca o nula participación en asuntos políticos). También se anexó la valoración de un grupo de expertos (investigadores que desarrollaron estudios en la zona maya de Quintana Roo). Para este último grupo, el ejercicio de valoración consistió en determinar, a partir de su conocimiento de la comunidad maya, cómo ésta valorará sus unidades de paisaje. Esto nos permitiría conocer en qué medida los expertos conocen las preferencias de los habitantes de la zona maya con respecto a las unidades de paisaje seleccionadas.

En la fase IV se llevó a cabo el análisis de los datos y la elaboración de las escalas de valores, en donde, de acuerdo con el método utilizado, las preferencias de los entrevistados reflejaron los valores no monetarios de las unidades de paisaje. En particular, a la escala de valores obtenida se le llamó escala de valores relativa, pues fue con relación a las seis unidades de paisaje evaluadas. Además, para poder llevar a cabo la comparación de escalas entre diferentes grupos, el método incluye, después del conteo de las preferencias (votos) y una vez que están ordenadas de mayor a menor, una estandarización de 0 a 100 para cada una (Dunn-Rankin, et al., 2004). Así, la similitud de la escala de un grupo fue evaluada con respecto a la obtenida para los otros grupos por medio del método de correlación por orden llamado coeficiente de correlación T Kendall's tau (Dunn-Rankin et al., 2004).

Una vez concluido este análisis, las escalas de valor relativo de cada grupo se presentaron en cada comunidad estudiada a través de talleres comunitarios, con el fin de tener una retroalimentación de los grupos comunitarios estudiados y de entender las razones de las preferencias de cada uno. Aunado a esto se seleccionaron dos informantes clave de cada comunidad y se les aplicó una entrevista a fondo, donde se abordó la importancia de las unidades de paisaje en cada ejido (Bernard, 2006).

Posteriormente, con la información obtenida en estas actividades se identificaron los valores de uso y de no uso de las unidades de paisaje a través del análisis cualitativo, que consistió en la clasificación de la información derivada de los talleres, de las entrevistas y de las observaciones realizadas en campo. La clasificación se realizó utilizando como base a Barbier (1994), Chuenpagdee (1998) y adecuando la tipología de valores presentada por Brown (2006), utilizando el software QSR N6. Así tam- 
bién, los servicios ecológicos y de bienestar de las unidades de paisaje se identificaron a través de la revisión bibliográfica de literatura reciente.

\subsection{Talleres comunitarios}

La parte ilustrativa o las fotos de los resultados anteriores con su respectiva escala se mostraron en las tres comunidades estudiadas durante siete talleres; dos se llevaron a cabo en Noh Cah, cuatro en Señor y uno en XHazil sur. En esta última comunidad se convocó a la gente a participar en cuatro talleres pero sólo asistió un día. Estas actividades se realizaron tomando en cuenta los usos y costumbres locales, por ello se convocó de forma separada a los hombres de las mujeres. No obstante, en ambos casos, se mostraron las dos escalas y, cuando las hubo, se preguntaron las razones de sus elecciones y a qué se debían las diferencias entre el orden de escalas de importancia de las unidades de paisaje de cada grupo de interés.

\section{Resultados}

\subsection{Prediagnóstico (delimitación y selección de las unidades de paisaje)}

El resultado de los mapas etnográficos para los tres ejidos de interés muestra que los espacios plasmados por los informantes clave fueron principalmente el monte alto o ya'ax k'aax, monte bajo o akalché, las zonas agrícolas donde destacan los acahuales o hubchés, la sabana, las lagunas, aguadas y cenotes; los poblados y los caminos (figuras 2, 3, y 4). Las delimitaciones realizadas por los informantes clave de las comunidades permitieron optar por una selección de espacios que la gente reconociera y a la cual tuviera acceso y que además contara con potencialidades productivas o de conservación.

Con base en lo anterior se seleccionaron seis espacios para la valoración no-monetaria: 1) monte alto o selva alta y mediana subperennifolia, 2) monte bajo o selva baja, 3) acahual de cinco a 10 años, 4) acahual de 20 o más años, 5) sabanas, y 6) cuerpos de agua. Los acahuales se dividieron en dos tipos porque, según las entrevistas realizadas a informantes clave y en la revisión de literatura, su uso de acuerdo con el periodo de descanso es diferenciado. En particular, se ha reportado el uso de los acahuales de cinco a 10 años principalmente para la milpa, sin embargo, si nos referimos al periodo óptimo de descanso en el sistema de roza tumba y quema, se encontró que era mayor de 20 años, condición de la que se obtiene otro tipo de productos tal como lo menciona BelloBaltazar (2001). 
Figura 2

Mapa etnográfico ejido Noh Cah

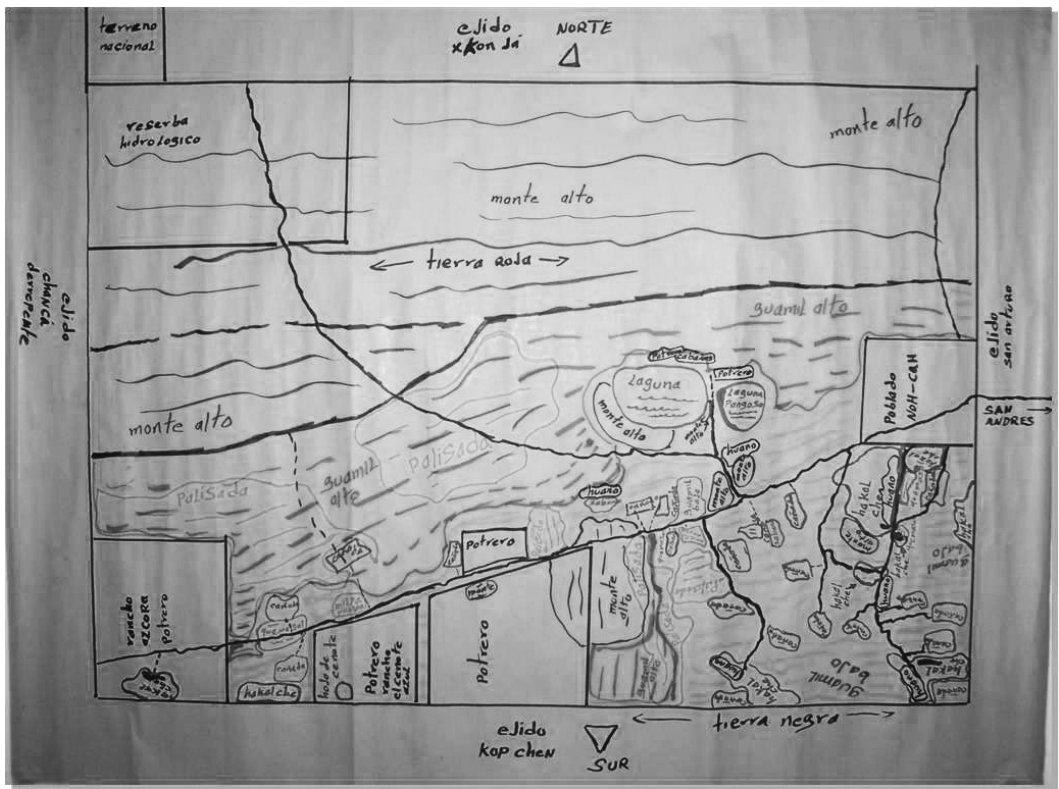

Fuente: elaborado por ejidatarios de la comunidad Noh Cah.

\section{Figura 3 \\ Mapa etnográfico del ejido X-Maben}

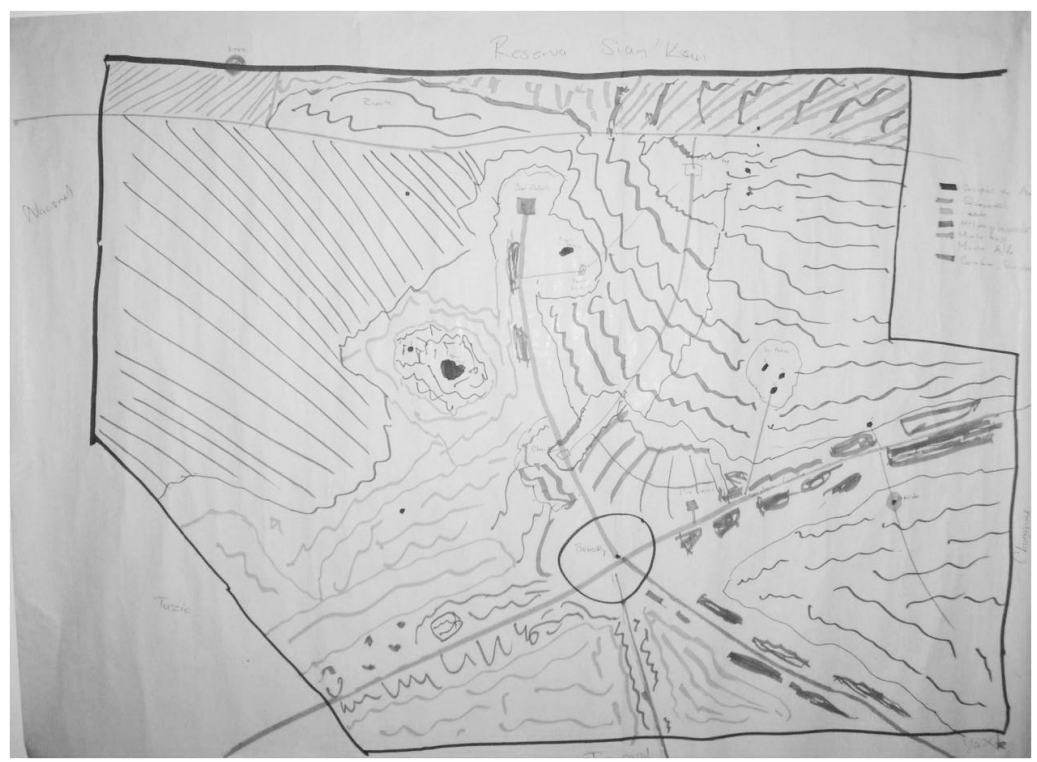

Fuente: elaborado por ejidatarios de la comunidad Señor. 


\section{Figura 4}

Mapa etnográfico del ejido X-Hazil sur y anexos

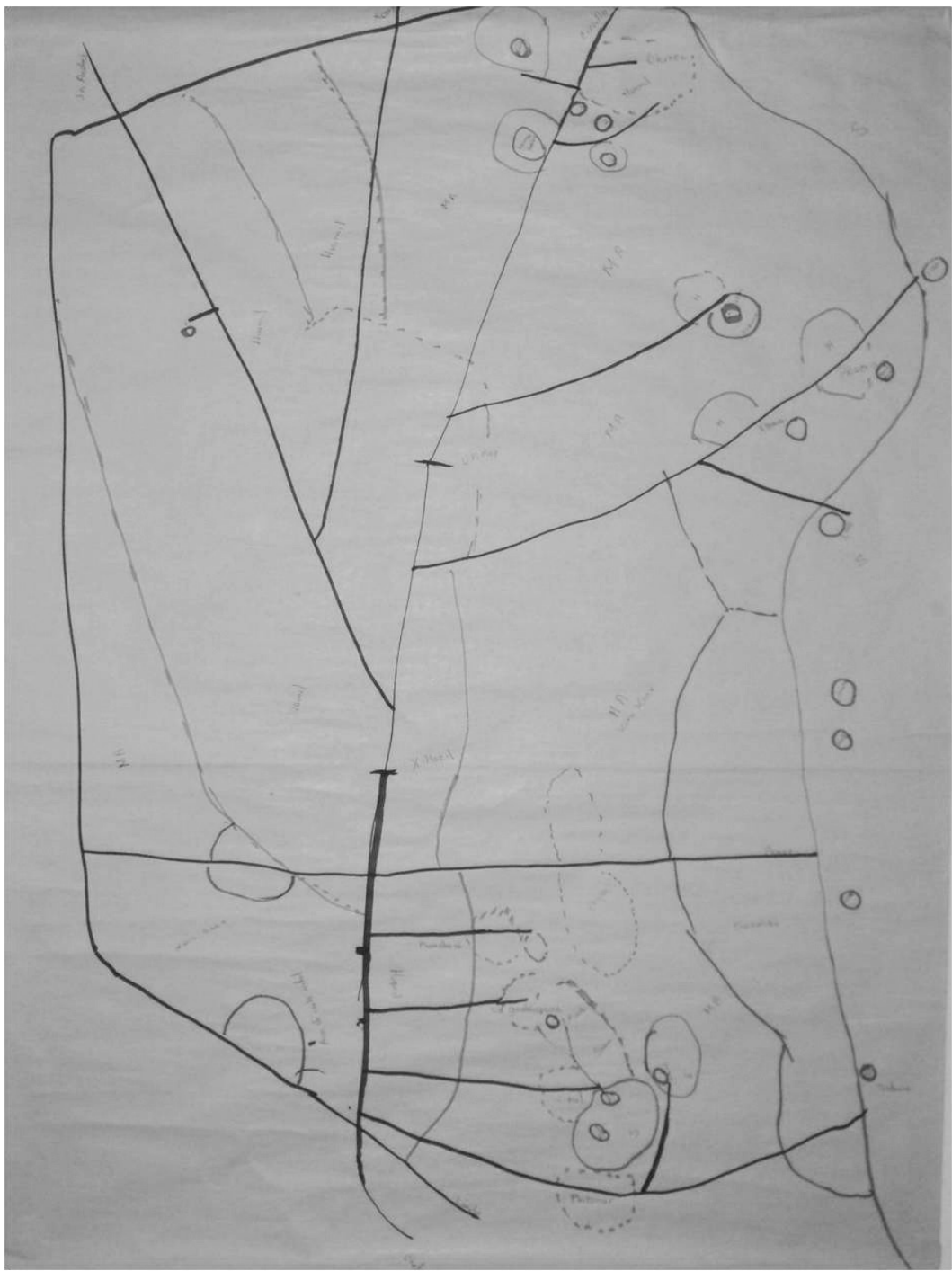

Fuente: elaborado por ejidatarios de la comunidad X-Hazil sur. 
Por otro lado, las lagunas, aguadas y cenotes se agruparon en la unidad de cuerpos de agua; esta decisión responde principalmente a su origen, que en todos los casos es caliza o cárstico (Elías-Gutiérrez et al., 2007) y al tipo de actividades que se realizan y que no son diferentes entre sí.

Finalmente, las sabanas se separaron de los cuerpos de agua porque no en todos los casos están asociadas a ellos y la actividad característica de estos espacios es la cacería (Santos-Fita et al., 2012). Por ejemplo, para el caso del ejido X-Hazil sur y anexos hay grandes extensiones de sabana entre la selva que para los cazadores representan sitios óptimos para su actividad.

En el cuadro 1 se presenta el resumen de las unidades de paisaje seleccionadas para realizar el ejercicio de valoración con nombre en espańol y en maya, así como las actividades y los productos que de cada unidad se obtienen actualmente, tal y como fueron mencionados durante la etapa de elaboración de los mapas, en los recorridos y también en la revisión de literatura.

\section{Cuadro 1}

Unidades de paisaje, actividades y productos actuales

\begin{tabular}{|c|c|c|}
\hline Unidad de paisaje & $\begin{array}{l}\text { Nombre en } \\
\text { maya-yucateco }\end{array}$ & Actividades y productos \\
\hline $\begin{array}{l}\text { Monte Alto } \\
\text { (Selva alta y mediana } \\
\text { Subperennifolia) }\end{array}$ & $\begin{array}{l}\text { Ya'ax K'aax, } \\
\text { Kana K'aax }\end{array}$ & $\begin{array}{l}\text { Cacería; aprovechamiento forestal } \\
\text { para maderas blandas y duras; chicle; } \\
\text { melífero, plantas medicinales, forraje. }\end{array}$ \\
\hline Monte Bajo (Selva baja) & Caba K'aax, Sakalche & $\begin{array}{l}\text { Cacería, palizada, melífero, huano, } \\
\text { leńa, plantas medicinales, forraje. }\end{array}$ \\
\hline Acahual de 20 años & Hubché & $\begin{array}{l}\text { Palizada, leña, milpa, huano, agro- } \\
\text { forestería, cultivos comerciales de } \\
\text { chile habanero, ganadería extensiva. }\end{array}$ \\
\hline $\begin{array}{l}\text { Acahual de cinco } \\
\text { a } 10 \text { ańos }\end{array}$ & Hubché & $\begin{array}{l}\text { Milpa, plantas medicinales, cultivos } \\
\text { comerciales de chile habanero, gana- } \\
\text { dería extensiva. }\end{array}$ \\
\hline Sabana & Chac k'aan* & Cacería \\
\hline $\begin{array}{l}\text { Cuerpos de agua } \\
\text { (Lagunas y cenotes) }\end{array}$ & & Pesca, nadar, turismo alternativo. \\
\hline
\end{tabular}

*Bello-Baltazar (2001) menciona que se asigna ese nombre a la sabana, sin embargo en las entrevistas realizadas a ese espacio le llaman sabana, igual que en el espańol.

Fuente: elaboración propia.

Cabe mencionar que con esas seis unidades de paisaje seleccionadas, el cuestionario utilizado estuvo formado por: 


$$
\begin{gathered}
\mathrm{N}=\mathrm{n}(\mathrm{n}-1) / 2 \\
=6(6-1) / 2 \\
=6(5) / 2=15 \text { preguntas }
\end{gathered}
$$

\subsection{Valoración no monetaria de las unidades de paisaje}

Las características de la población entrevistada se presentan en el cuadro 2. En Noh Cah, debido a que la población es pequeña (19 hogares) en comparación con las otras comunidades, el grupo de ejidatarios se agrupó con el de no ejidatarios. Como se mencionó con anterioridad, la escala obtenida -de mayor a menor importancia- representa los valores no-monetarios de las unidades de paisaje seleccionadas. Aunque elegir el grupo que diera la base para acomodar el orden de las escalas de los otros grupos pudiera hacerse de una manera un tanto arbitraria, el orden que se consideró como base para ordenar las escalas de los demás grupos analizados fue el de ejidatarios, porque ellos son los que cuentan con los derechos legales sobre el uso de las tierras ejidales. En las escalas obtenidas, las unidades de paisaje se agruparon naturalmente de acuerdo con tres categorías de importancia que fueron alta, media y baja.

\section{Cuadro 2}

\section{Demografía de los participantes}

\begin{tabular}{llrrrr}
\hline Comunidad & \multicolumn{1}{c}{$\begin{array}{c}\text { Grupo } \\
\text { Noh Cah }\end{array}$} & $\begin{array}{c}\text { Promedio de edad } \\
\text { (Años) }\end{array}$ & S.D. ${ }^{*}$ & $\begin{array}{c}\text { Intervalo } \\
\text { (Años) }\end{array}$ & $n^{* *}$ \\
& Ejidatarios & 49.46 & 11.53 & $36-70$ & 14 \\
& No-ejidatarios & 33.5 & 3.11 & $30-37$ & 4 \\
\multirow{5}{*}{ Señor } & Mujeres & 37.23 & 18.78 & $16-71$ & 14 \\
& Ejidatarios & 49.93 & 9.77 & $30-73$ & 27 \\
& No-ejidatarios & 28.97 & 7.88 & $16-46$ & 30 \\
\multirow{3}{*}{ Xhazil-Sur } & Mujeres & 38.85 & 9.78 & $24-59$ & 26 \\
& Ejidatarios & 51.24 & 14.08 & $29-86$ & 25 \\
& No-ejidatarios & 28.53 & 11.94 & $16-70$ & 26 \\
& Mujeres & 38.2 & 12.7 & $16-70$ & 29 \\
Total & Expertos & 43.55 & 8.1 & $29-56$ & 20 \\
\hline \multirow{2}{*}{ S.D. } & & & & 215 \\
\hline
\end{tabular}

* S.D. = desviación estándar $\mathrm{n}^{* *}=$ Número total de entrevistados Fuente: elaboración propia. 
En la escala para el grupo de los hombres de Noh Cah, las unidades de paisaje que forman el primer grupo de valor, el de mayor importancia, fue el monte alto y el acahual de 20 años o más; el segundo grupo de valor o de importancia intermedia estuvo compuesto por el acahual de cinco a 10 ańos, el monte bajo y por los cuerpos de agua. El último grupo de valor o de importancia baja lo ocupó la sabana.

Respecto a las preferencias del grupo de las mujeres, el acahual de 20 años o más y el monte alto formaron el grupo de importancia alta, mientras que los cuerpos de agua, el monte bajo y el acahual de cinco a 10 años fue el grupo de importancia intermedia y la sabana fue la unidad de paisaje de importancia baja (cuadro 3). El análisis de la correlación por orden en las escalas de hombres y mujeres no fue significativo (cuadro 4). Es decir, no hubo correlación o similitud entre éstas y por lo tanto se presentan dos escalas gráficas, una para cada grupo (figuras 5 y 6). Se puede decir que son dos escalas de valoración diferentes, una para los hombres y otra para las mujeres, y es por esta razón que se presentan dos escalas gráficas correspondientes a cada grupo.

\section{Cuadro 3}

\section{Escalas de importancia de las unidades de paisaje para Noh Cah}

\begin{tabular}{lcc}
\hline \multicolumn{1}{c}{ Escenario de daño } & Hombres & Mujeres \\
\hline Daño al monte alto & $84(1)$ & $71(2)$ \\
Daño al acahual de 20 años o más & $68(2)$ & $78(1)$ \\
Daño al acahual de cinco a 10 años & $47(3)$ & $42(5)$ \\
Daño al monte bajo & $44(4)$ & $45(4)$ \\
Daño a los cuerpos de agua & $42(5)$ & $46(3)$ \\
Daño a la sabana & $14(6)$ & $18(6)$ \\
\hline
\end{tabular}

Para cada grupo analizado se observan dos escalas, la primera en número en escala del 0 al 100 y del lado derecho se presentan los resultados pero en escala de orden, del 1 al 6 . Fuente: elaboración propia.

\section{Cuadro 4}

Resultados de la correlación por orden para la comunidad Noh Cah

\begin{tabular}{lcc}
\hline \multicolumn{1}{c}{ Grupo de interés } & Hombres & Mujeres \\
\hline Hombres & 1.000 & 0.600 \\
Mujeres & & 1.000 \\
\hline
\end{tabular}

Fuente: elaboración propia. 
Figura 5

Escala de valor relativo de las unidades de paisaje de los hombres de Noh Cah

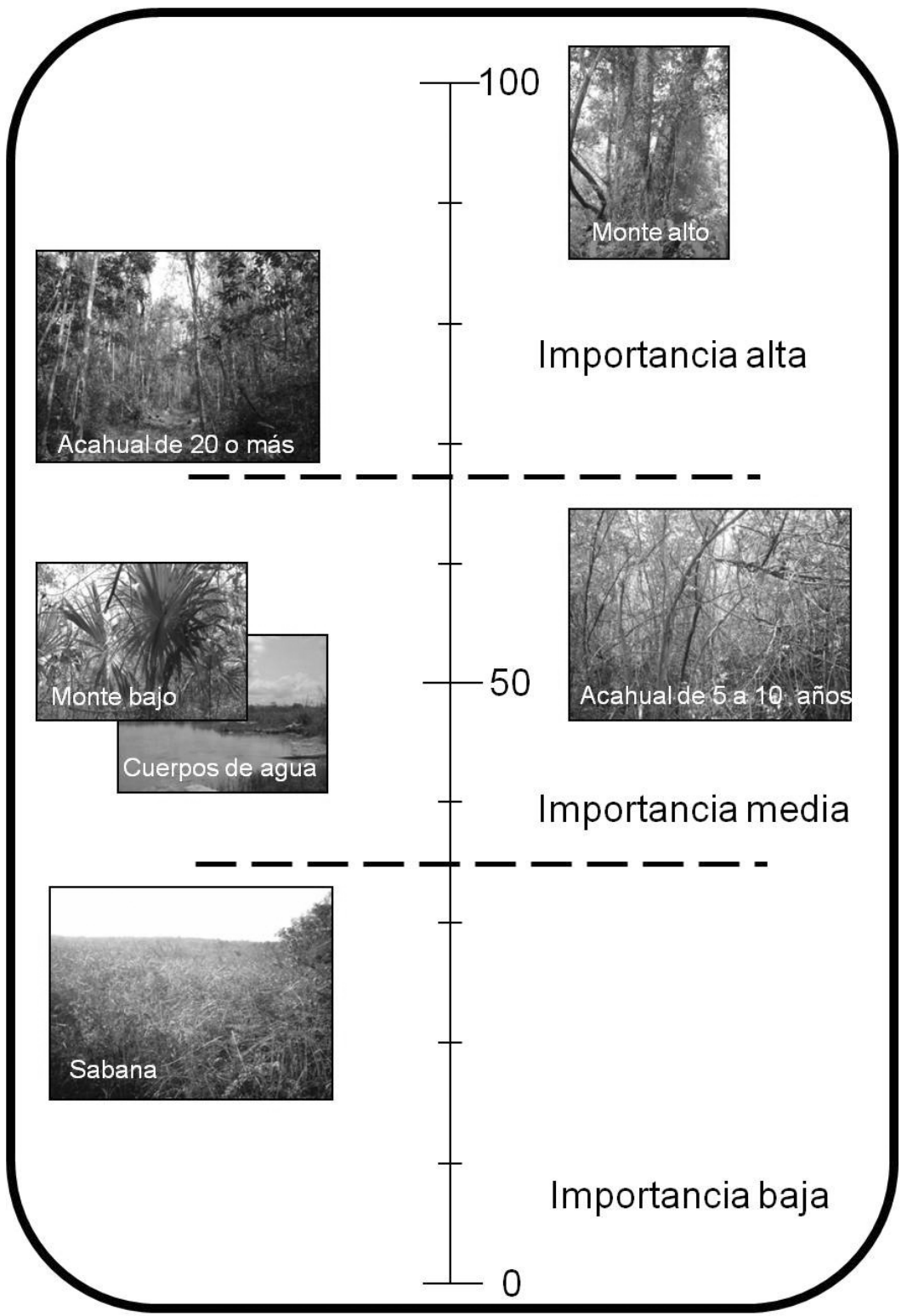

Fuente: elaboración propia. 


\section{Figura 6}

Escala de valor relativo de las unidades de paisaje de las mujeres de Noh Cah

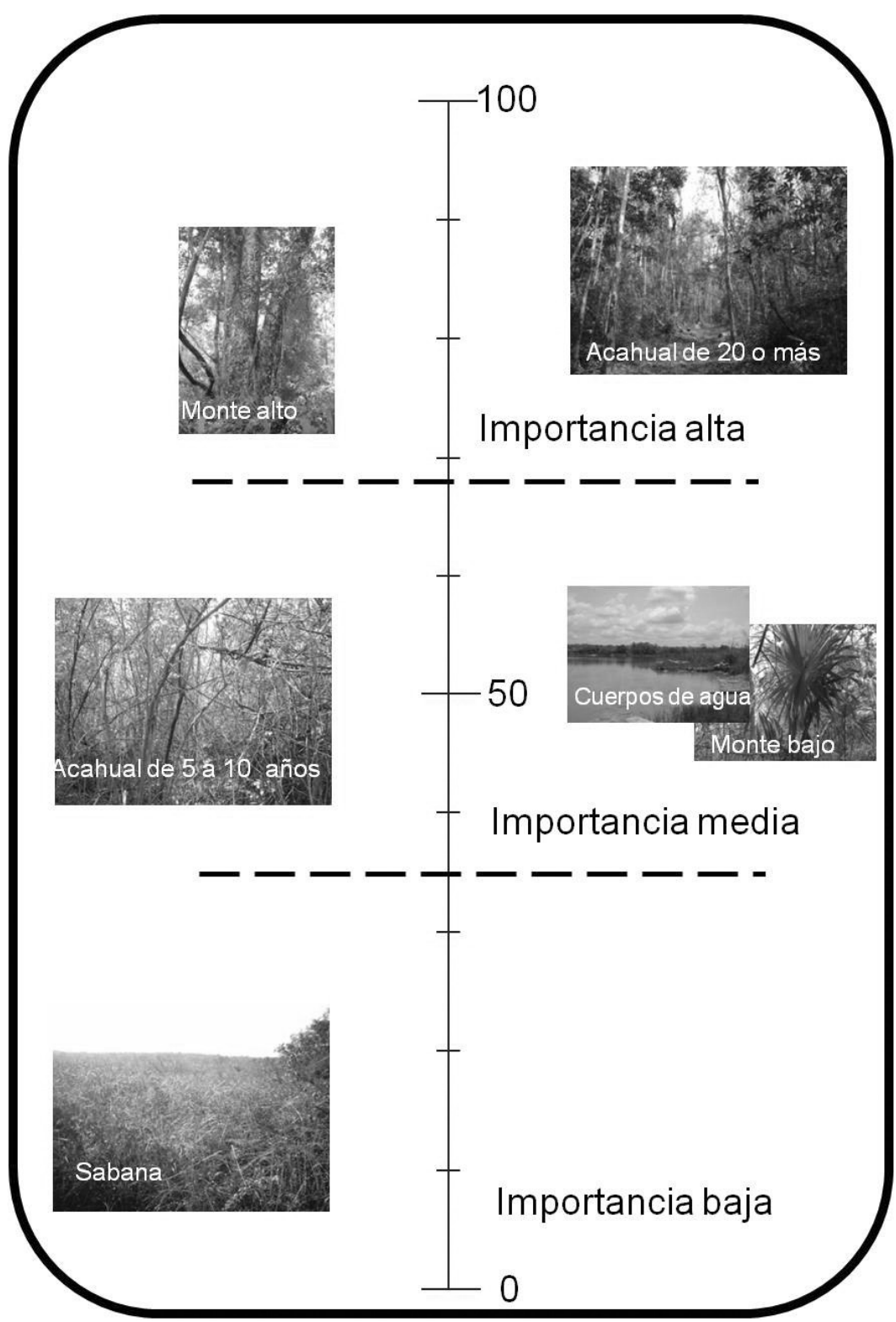

Fuente: elaboración propia. 
Para la comunidad Señor, las escalas obtenidas por cada grupo se presentan en el cuadro 5 . El grupo de unidades de mayor importancia para ejidatarios incluyó el monte alto y el acahual de 20 o más años, el grupo de valor intermedio correspondió al acahual de cinco a 10 años, al monte bajo y a los cuerpos de agua, mientras que el valor bajo sólo incluyó a la sabana. Este resultado fue similar para la escala de valoración de los no ejidatarios, donde tiene mayor importancia al monte alto y al acahual de 20 o más años, el grupo de valor intermedio fue para los cuerpos de agua, el monte bajo y el acahual de cinco a 10 ańos, mientras que el grupo de valor bajo fue para la sabana.

Cuadro 5

Escalas de importancia de las unidades de paisaje para la comunidad Señor

\begin{tabular}{lrrr}
\hline \multicolumn{1}{c}{ Escenario de daño } & Ejidatarios & No ejidatarios & \multicolumn{1}{c}{ Mujeres } \\
\hline Daño al monte alto & $87(1)$ & $80(1)$ & $69(2)$ \\
Daño al acahual de 20 años o más & $74(2)$ & $73(2)$ & $71(1)$ \\
Daño al acahual de cinco a 10 años & $48(3)$ & $49(4)$ & $43(5)$ \\
Daño al monte bajo & $44(4)$ & $41(5)$ & $54(3)$ \\
Daño a los cuerpos de agua & $42(5)$ & $51(3)$ & $51(4)$ \\
Daño a la sabana & $17(6)$ & $7(6)$ & $12(6)$ \\
\hline
\end{tabular}

Fuente: elaboración propia.

Para las mujeres de Señor, el grupo de valor alto estuvo representado por el acahual de 20 o más años y el monte alto; el grupo de valor intermedio fue para el monte bajo, así como para los cuerpos de agua y el acahual de cinco a 10 años, mientras que la sabana quedó en el grupo de valor bajo. Derivado de lo anterior, podemos observar que hubo algunas diferencias, aunque sutiles, con respecto al orden de las unidades entre hombres y mujeres; por ejemplo, la ubicación de los cuerpos de agua que fueron más importantes para los no ejidatarios y para las mujeres pero menos importantes para los ejidatarios. Sin embargo, la prueba de correlación por orden (cuadro 6) muestra que la escala de ejidatarios y no ejidatarios fueron similares, mientras que la de mujeres no lo fue con respecto a los otros dos grupos. Por esta razón se decidió hacer dos escalas de importancia, una para hombres (incluye a ejidatarios y a no ejidatarios) y otra para mujeres (figuras 7 y 8 ). 


\section{Figura 7}

Escala de valor relativo de las unidades de paisaje de los hombres de la comunidad Señor

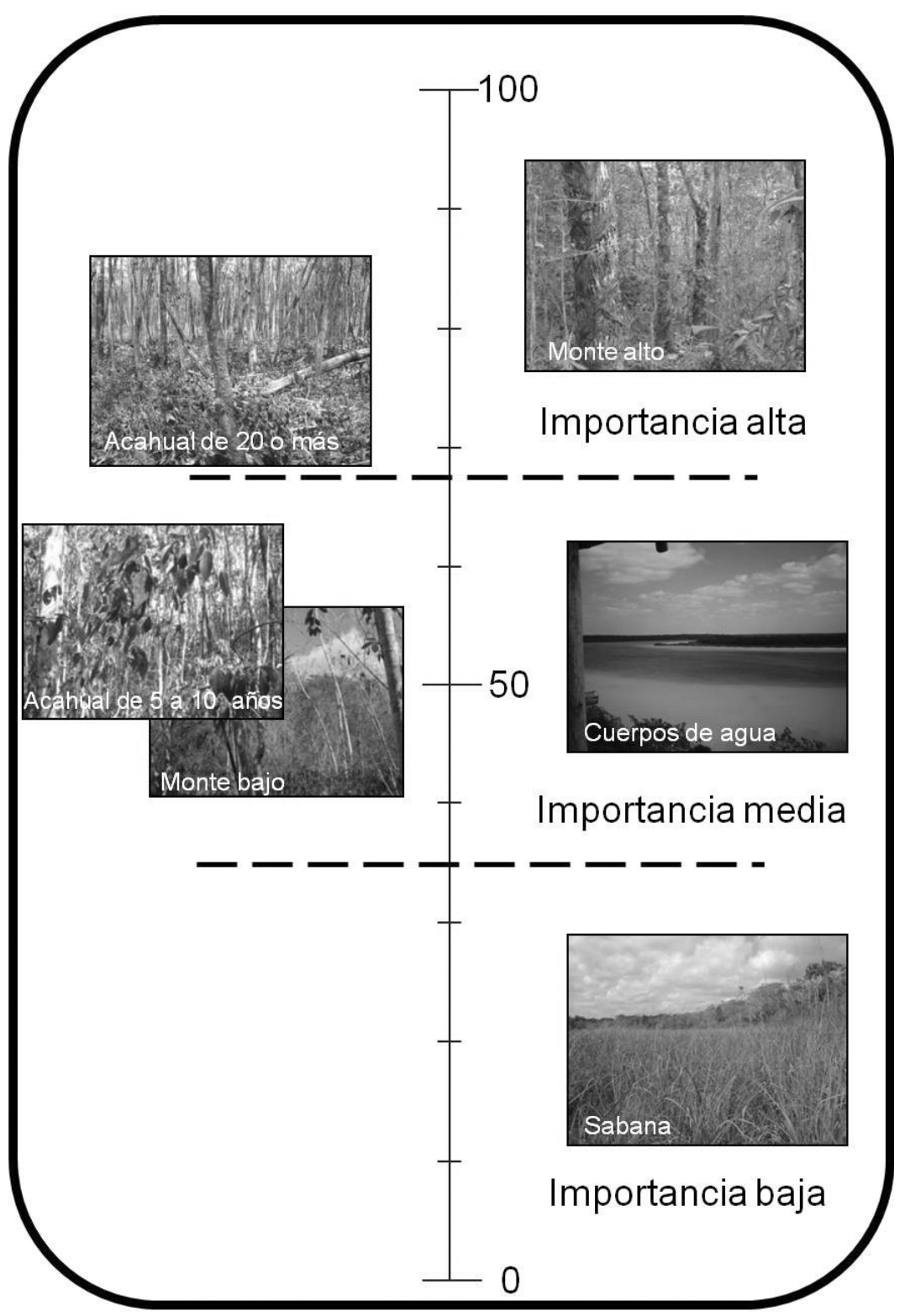

Fuente: elaboración propia. 
Figura 8

Escala de valor relativo de las unidades de paisaje de las mujeres de la comunidad Seńor

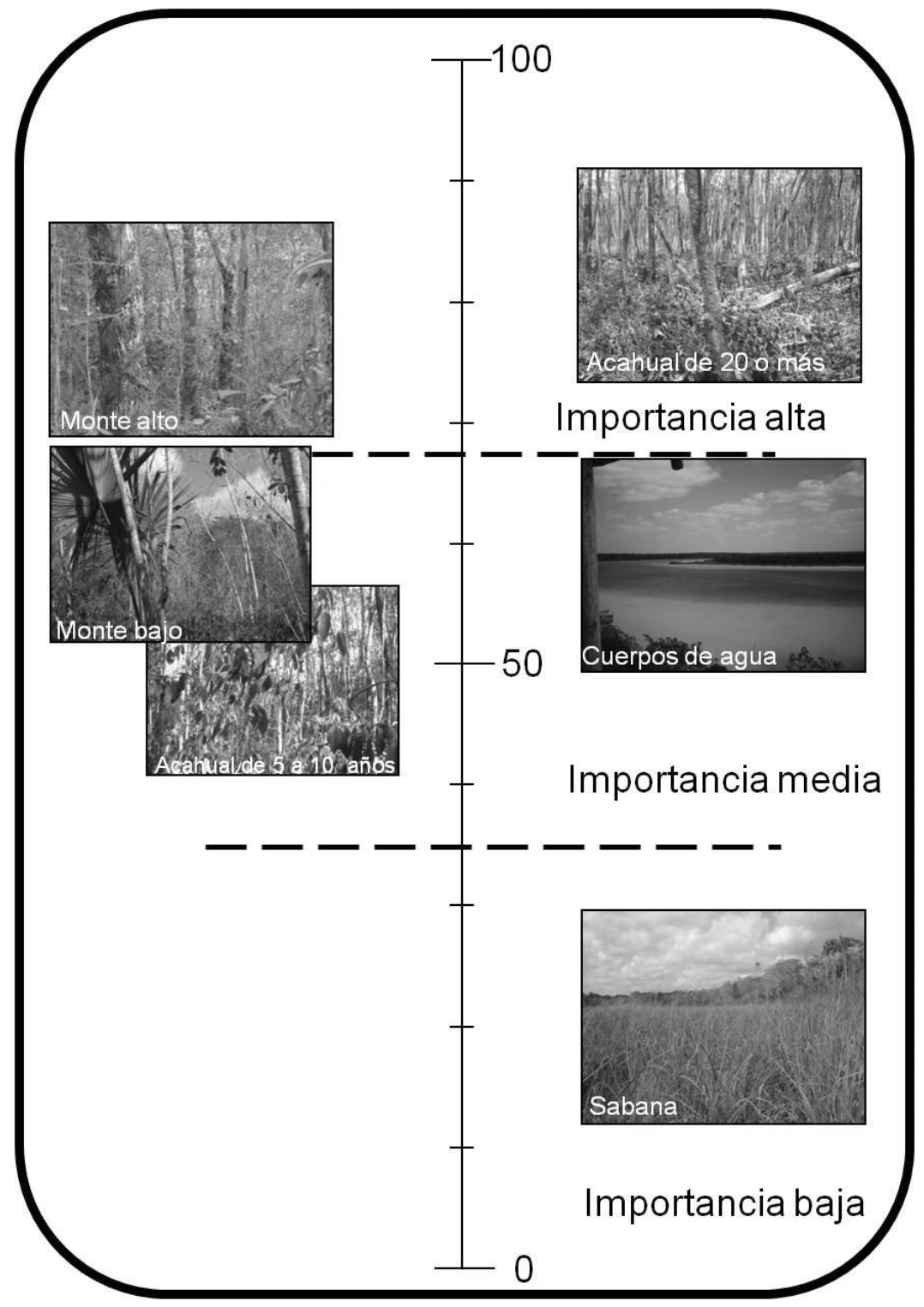

Fuente: elaboración propia. 


\section{Cuadro 6 \\ Resultados de la correlación por orden para la comunidad Señor por grupo de interés}

\begin{tabular}{lrrr}
\hline \multicolumn{1}{c}{ Grupo de interés } & Ejidatarios & No ejidatarios & \multicolumn{1}{c}{ Mujeres } \\
\hline Ejidatarios & 1.000 & $0.733^{*}$ & 0.600 \\
No ejidatarios & & 1.000 & 0.600 \\
Mujeres & & & 1.000 \\
\hline
\end{tabular}

*La correlación es significativa al nivel 0.05 (bilateral).

Fuente: elaboración propia.

Para la comunidad X-Hazil sur las escalas obtenidas se presentan en el cuadro 7. Aquí se puede apreciar que para los tres grupos comunitarios de interés, el monte alto fue la unidad más importante y la sabana la menos importante. De manera específica, para los ejidatarios, el grupo de valor alto de las unidades estuvo dado sólo por el monte alto y, el grupo de valor intermedio incluyó el acahual de 20 años o más; el monte bajo, el acahual de cinco a 10 años y los cuerpos de agua, mientras que la sabana correspondió al grupo de valor bajo. El orden de importancia de las unidades de paisaje para los no ejidatarios y para las mujeres fue el mismo; en ambos casos, el monte alto y el acahual de 20 o más años fueron de importancia alta, mientras que los cuerpos de agua, el monte bajo y el acahual de cinco a 10 años fueron de importancia intermedia y la sabana de importancia baja. Para X-Hazil sur, los resultados del análisis estadístico para los diferentes grupos se correlacionaron significativamente, es decir, fueron escalas de valoración similares (cuadro 8), por esta razón, sólo se presenta una escala gráfica de valores, que en este caso representa las preferencias de los tres grupos comunitarios (figura 9).

\section{Cuadro 7}

Escalas de importancia de las unidades de paisaje para la comunidad X-Hazil Sur

\begin{tabular}{lccc}
\hline \multicolumn{1}{c}{ Escenario de daño } & Ejidatarios & No ejidatarios & Mujeres \\
\hline Daño al monte alto & $77(1)$ & $72(1)$ & $75(1)$ \\
Daño al acahual de 20 años o más & $69(2)$ & $64(2)$ & $57(2)$ \\
Daño al monte bajo & $59(3)$ & $48(4)$ & $53(4)$ \\
Daño al acahual de cinco a 10 años & $53(4)$ & $45(5)$ & $43(5)$ \\
Daño a los cuerpos de agua & $38(5)$ & $51(3)$ & $56(3)$ \\
Daño a la sabana & $4(6)$ & $20(6)$ & $15(6)$ \\
\hline
\end{tabular}

Fuente: elaboración propia. 


\section{Cuadro 8}

Correlación por orden de la prueba Kendall's tau por grupo de interés de X-Hazil sur

\begin{tabular}{lrrr}
\hline \multicolumn{1}{c}{ Grupo de interés } & Ejidatarios & No-ejidatarios & \multicolumn{1}{c}{ Mujeres } \\
\hline Ejidatarios & 1.000 & $0.733^{*}$ & $0.733^{*}$ \\
No-ejidatarios & & 1.000 & 1.000 \\
Mujeres & & & 1.000 \\
\hline
\end{tabular}

*La correlación es significativa al nivel 0.05 (bilateral)

Fuente: elaboración propia.

Con respecto a la escala de valores del grupo de expertos académicos, el monte alto fue la unidad de importancia alta, los cuerpos de agua, el acahual de 20 años o más, el monte bajo y el acahual de cinco a 10 años, formaron el grupo de valor intermedio, mientras que la sabana ocupó el valor bajo (cuadro 9). En la figura 10 se presenta su escala gráfica. En la comparación de la escala de valores relativos de los expertos con los grupos de cada comunidad, sólo se observaron diferencias significativas con la escala de hombres de Noh Cah (cuadro 10).

\section{Cuadro 9}

\section{Escala de valor relativo del grupo de expertos}

\begin{tabular}{lc}
\hline \multicolumn{1}{c}{ Escenario de daño } & Expertos \\
\hline Daño al monte alto & $84(1)$ \\
Daño al acahual de 20 años o más & $62(2)$ \\
Daño al monte bajo & $40(4)$ \\
Daño al acahual de 5 a 10 años & $38(5)$ \\
& \\
Daño a los cuerpos de agua & $63(3)$ \\
Daño a la sabana & $13(6)$ \\
\hline
\end{tabular}

Fuente: elaboración propia. 


\section{Figura 9}

Escala gráfica de valores relativos de las unidades de paisaje para la comunidad de X-Hazil sur

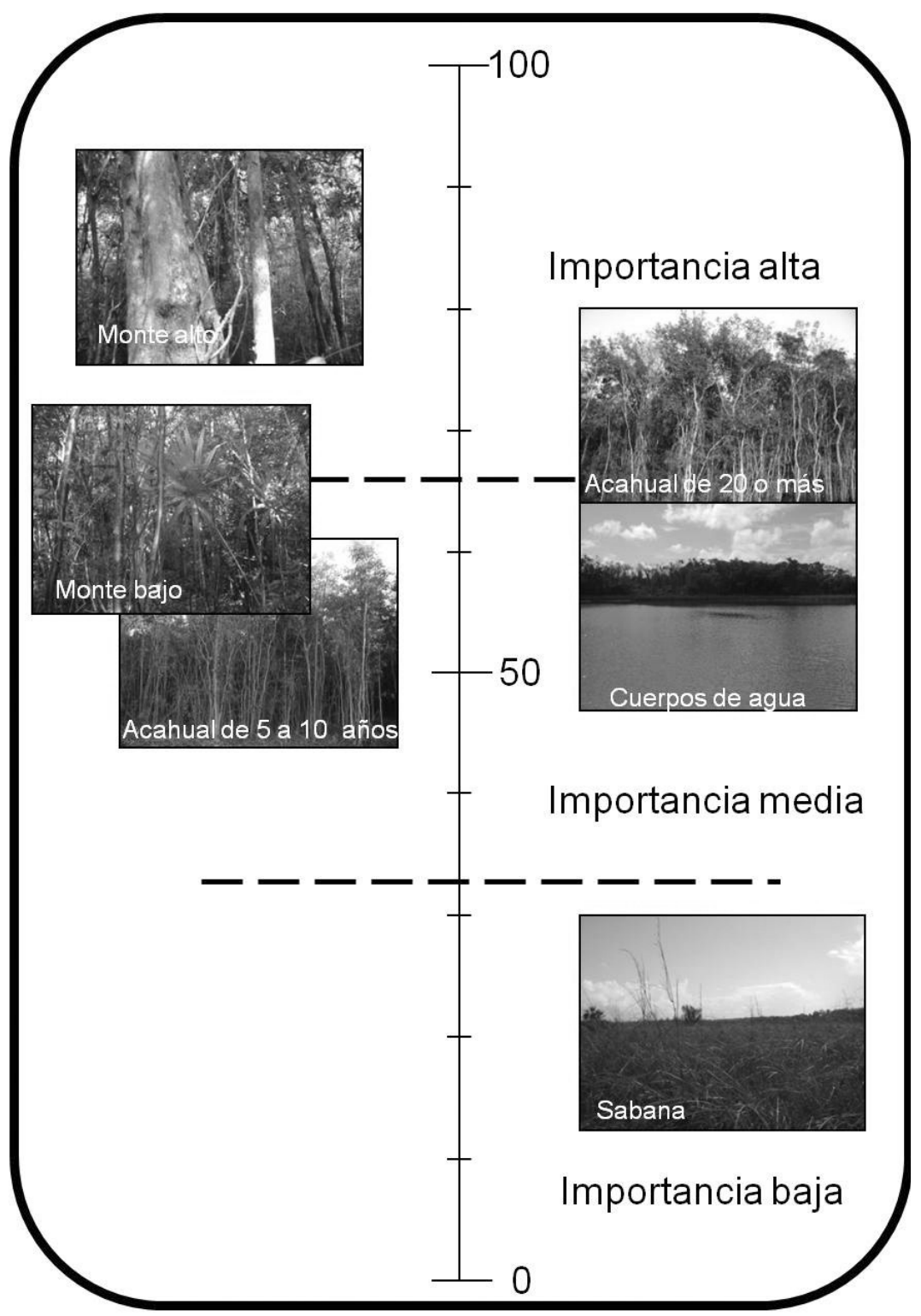

Fuente: elaboración propia. 


\section{Cuadro 10 \\ Comparación entre la escala de expertos y los grupos de interés de cada comunidad con la prueba Kendall's tau}

\begin{tabular}{lcccccc}
\hline Grupo de interés & $E$ & $H N$ & $M N$ & $H S$ & $M S$ & $X H$ \\
\hline $\mathrm{E}$ & 1 & 0.6 & $0.867^{*}$ & $0.733^{*}$ & $0.733^{*}$ & $0.867^{*}$ \\
\hline
\end{tabular}

*La correlación es significativa al nivel 0.05 (bilateral)

$\mathrm{E}=$ Expertos, $\mathrm{HN}=$ Hombres Noh Cah, MN=Mujeres Noh Cah, HS=Hombres Señor, MS=Mujeres Seńor, $\mathrm{XH}=\mathrm{X}-\mathrm{Hazil}$ sur.

Fuente: elaboración propia.

\subsection{Talleres comunitarios}

En los talleres comunitarios para las tres comunidades, los hombres y las mujeres se mostraron de acuerdo con el orden de las escalas de valoración presentado, aunque también identificaron las diferencias encontradas entre hombres y mujeres. Estas diferencias las atribuyeron principalmente a que los hombres conocen bien el monte y, en general, el ejido, mientras que las mujeres se dedican más a las labores del hogar. Por otra parte, con respecto a las razones del orden de preferencias de las unidades mencionaron que el monte alto y los acahuales de 20 años o más son los más importantes, principalmente, porque allí es donde la milpa se cultiva mejor; donde encuentran maderas grandes para la construcción de las casas, así como los animales para la cacería, y donde también se puede extraer miel silvestre.

Siguiendo sus explicaciones para el grupo de valor intermedio, en este caso para el monte bajo, los cuerpos de agua y el acahual de cinco a 10 ańos, mencionaron que en el monte bajo los suelos no son muy buenos para la milpa, y lo que se produce es limitado; que son sitios donde se extrae el huano botan (Sabal yapa), el huano kum (Crysophila argéntea) ${ }^{l}$ pero que si no se tiene el permiso de aprovechamiento, los precios de venta son muy bajos. Además, para los cuerpos de agua mencionaron que es vital para los animales, y la actividad que allí se realiza es la pesca principalmente para autoconsumo. Los acahuales de cinco a 10 años -a pesar de ser los que en la actualidad se utilizan más para la milpa-mencionaron que como son muy jóvenes su productividad no es muy buena y que llegan a requerir insumos externos como fertilizantes o herbicidas para tener una producción aceptable; sin embargo, reconocieron que la calidad de sus productos ya no es la misma. Finalmente, las sabanas que fueron de

\footnotetext{
${ }^{1}$ El huano botan (Sabal yapa), el huano kum (Crysophila argentea) tiene múltiples usos en la zona maya, los más relevantes son para la construcción de los techos de las casas y en la cocina para la preparación de algunos alimentos.
} 
Figura 10

Escala de valores relativos del grupo de expertos en la zona maya

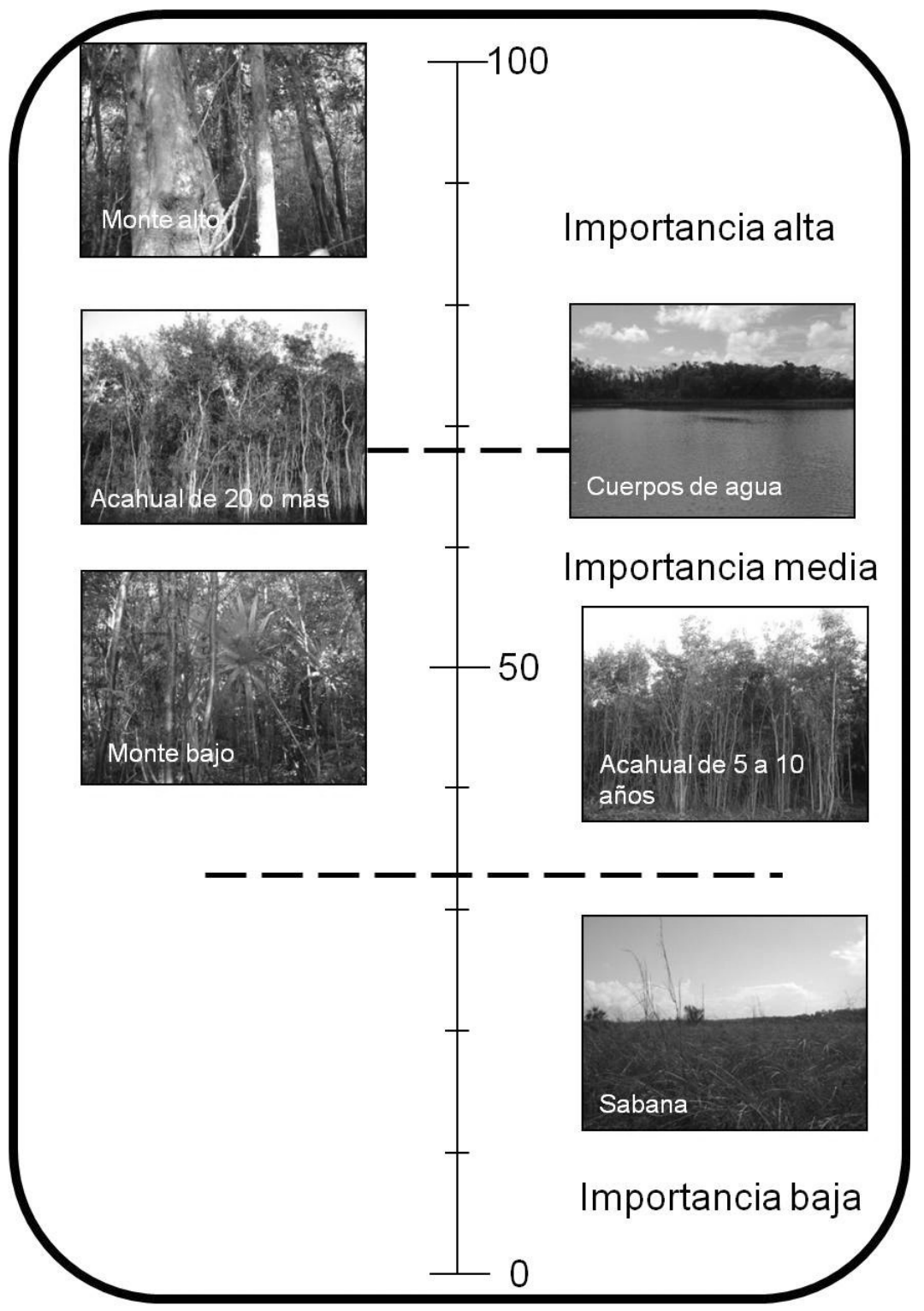

Fuente: elaboración propia. 
importancia baja, mencionaron que se debe a que allí no se da la milpa y que por lo tanto no hay muchos productos aprovechables. Algunos hombres cazadores reconocieron que estos sitios son buenos para la cacería pero como son lugares muy alejados no toda la gente los conoce.

\subsection{Valores de uso y no uso de las unidades de paisaje}

El cuadro 11 presenta un resumen de los valores de uso y de no uso identificados en las unidades de paisaje utilizadas en este estudio. Los principales valores de uso directo identificados fueron de dos tipos, el valor de sustento-doméstico y el valor económico; el primero tiene que ver con la utilización de los espacios con el fin de satisfacer necesidades de autoconsumo, que no implican un intercambio monetario, como el caso de la cacería, la milpa, la colecta de plantas medicinales, los productos maderables para la construcción de casas, entre otros. El valor económico se relaciona con el uso de los espacios para extracción de productos que les generen un ingreso económico tales como la venta de maderas preciosas, venta de miel y otros productos no maderables como la hoja de palma de huano. Los valores de uso indirecto son los valores ecológico y biológico de las unidades de paisaje seleccionadas. Estos se identificaron a lo largo de todo el trabajo de campo ya que la gente mencionaba clasificaciones locales de las plantas o animales de cada espacio, así como asociaciones entre animales y plantas para desarrollar estrategias de aprovechamiento. Por ejemplo, para la cacería, mencionaban que los sitios ideales para cazar eran donde disponibilidad de agua y alimento para los animales como árboles frutales.

Los valores de no uso identificados fueron el de opción y el de existencia; el primero se refiere al potencial recreativo de un lugar, pero también al valor estético que se relaciona con la belleza paisajística, la cual se reconoce principalmente en el monte alto y en algunos cuerpos de agua como la Laguna Azul en el ejido X-Maben. El valor de no uso o de existencia se relaciona con el valor histórico-cultural reflejado en la realización de prácticas sociales características de la cultura maya como son los rezos y las ofrendas cuando hacían milpa en el monte alto o cuando van de cacería. Este valor también se puede relacionar con la creencia maya de la existencia de entes cuidadores del monte, de los cuales mencionaron a los aluxes, el Juan de monte, entre otros. También identificaron el valor a futuro al reconocer la importancia de mantener estos espacios en buenas condiciones para que lo disfruten los hijos. 


\section{Cuadro 11}

Valores de uso y de no uso identificados en las unidades de paisaje

\begin{tabular}{|c|c|c|c|}
\hline \multicolumn{2}{|c|}{ Valores de uso } & \multicolumn{2}{|c|}{ Valores de no uso } \\
\hline Directo & Indirecto & De opción & De existencia \\
\hline $\begin{array}{l}\text { Económico de sub- } \\
\text { sistencia (cacería, } \\
\text { plantas medicinales, } \\
\text { entre otros usos) de } \\
\text { venta en mercado } \\
\text { nacional o interna- } \\
\text { cional de maderas }\end{array}$ & $\begin{array}{l}\text { Biológico (reconoci- } \\
\text { miento e identifica- } \\
\text { ción de especies } \\
\text { animales y vegetales). }\end{array}$ & $\begin{array}{l}\text { Recreación (el sitio } \\
\text { con fines de } \\
\text { descanso). }\end{array}$ & $\begin{array}{l}\text { Histórico-cultural } \\
\text { (existencia de entes } \\
\text { cuidadores del mon- } \\
\text { te, así como la reali- } \\
\text { zación de prácticas } \\
\text { que caracterizan su } \\
\text { cultura). }\end{array}$ \\
\hline $\begin{array}{l}\text { preciosas, de miel y } \\
\text { otros productos no } \\
\text { maderables. }\end{array}$ & $\begin{array}{l}\text { Ecológico (recono- } \\
\text { cimiento e identifi- } \\
\text { cación de funciones } \\
\text { que desempeñan } \\
\text { algunas plantas y } \\
\text { animales en la selva, } \\
\text { la sabana y los cuer- } \\
\text { pos de agua). }\end{array}$ & $\begin{array}{l}\text { Estético (deleite y } \\
\text { disfrute del sitio, } \\
\text { belleza paisajística). }\end{array}$ & $\begin{array}{l}\text { A futuro (reconocer } \\
\text { la importancia de } \\
\text { estos sitios para las } \\
\text { generaciones veni- } \\
\text { deras). }\end{array}$ \\
\hline
\end{tabular}

Fuente: elaboración propia con base en la clasificación expuesta en Barbier (1994) y Chuenpagdee (1998).

\subsection{Servicios ecológicos y de bienestar que prestan las unidades de paisaje}

Durante las entrevistas, aplicación de cuestionarios y talleres, la gente mencionó lo que ellos perciben como beneficios (lo que en términos académicos se llama servicios ecológicos y de bienestar) que les brindan sus espacios productivos unidades de paisaje, delimitados en cada ejido, las cuales se resumen en el cuadro 12. Por ejemplo, el monte alto y el hubché de 20 años o más fueron los espacios donde la gente identificó mayores beneficios. En particular, mencionaron que es importante mantener el monte alto alrededor de la comunidad a manera de protección contra los huracanes. Además, reconocieron que los árboles les dan oxígeno, sombra y madera para construir sus casas, es decir, que son un elemento de gran importancia en estas unidades de paisaje. Así también, para la unidad que comprende al monte bajo resaltaron el aprovechamiento del huano y los bejucos para construir las casas o para hacer artesanías, respectivamente. Para el hubché de cinco a 10 años mencionaron que les proporciona leña, elemento necesario para cocinar diariamente. Aquí también mencionaron la formación del suelo, que es un proceso que mejora, por ejemplo, mientras más tiempo se deje de descanso al hubché. Para los cuerpos de agua mencionaron que el agua es vida y varios resal- 
taron que las lagunas y cenotes también tienen potencial turístico. Finalmente, mencionaron que la sabana proporciona agua a los animales del monte, ya que es un sitio que en temporada de lluvias se mantiene inundado.

\section{Cuadro 12}

Servicios ecológicos identificados por las comunidades estudiadas

\begin{tabular}{ll}
\hline \multicolumn{1}{c}{ Unidad de paisaje } & \multicolumn{1}{c}{ Servicio ecológico y de bienestar } \\
\hline $\begin{array}{l}\text { Monte alto (Kana Ka’ax, Ya’ax } \\
\text { Ka’ax) }\end{array}$ & $\begin{array}{l}\text { Protección contra los huracanes, oxígeno, alimento, } \\
\text { sombra, madera para la construcción de casas, } \\
\text { potencial para senderismo. }\end{array}$ \\
Monte bajo (kaba ka’ax) & $\begin{array}{l}\text { Material para construcción de las casas y artesanías, } \\
\text { oxígeno. }\end{array}$ \\
Hubché de cinco a 10 años & $\begin{array}{l}\text { Leña para cocinar, va ayudando al suelo a agarrar } \\
\text { fuerza para hacer la milpa (la formación del suelo). }\end{array}$ \\
Hubché de 20 años o más & $\begin{array}{l}\text { Oxígeno, protegen contra los huracanes, mejoran } \\
\text { el suelo para hacer la milpa después (la formación } \\
\text { del suelo). }\end{array}$ \\
Cuerpos de agua & $\begin{array}{l}\text { El agua es la vida, para quitar el calor, alimento, } \\
\text { potencial para turismo. }\end{array}$ \\
Sabana & Agua para los animales de la selva. \\
\hline
\end{tabular}

Fuente: elaboración propia.

\section{Discusión}

\subsection{Delimitación de las unidades de paisaje}

El paisaje actual de la zona maya de Quintana Roo es la expresión de la implementación de estrategias de uso múltiple de los recursos naturales que responde a una racionalidad tanto ecológica como económica (Toledo et al., 2008). Jiménez-Osornio et al. (2003) denominan a este proceso de adecuación tradicionalismo moderno. Esta diversidad de actividades las llevan a cabo dentro y fuera de sus comunidades, es decir, a través del manejo de la selva y también migrando a otras comunidades o municipios donde son prestadores de servicios principalmente en la zona hotelera de Tulúm o de Playa del Carmen (Santos-Fita et al., 2012).

Toledo et al. (2008) resaltan que los mosaicos de paisaje presentes en la zona maya de la península de Yucatán son el resultado tangible del mantenimiento, uso y manejo de la geo-agrodiversidad y de las masas de vegetación en donde los productores realizan un complejo manejo de unidades reconocidas y definidas con base en criterios de la vegetación, 
los suelos, las estaciones climáticas y el relieve, por medio del cual buscarán la optimización de su esfuerzo en el espacio y el tiempo. El mosaico de vegetación en diferentes estados sucesionales se puede atribuir principalmente a la agricultura tradicional de roza tumba y quema que tiene uso histórico por los mayas.

En este sentido, los resultados de las herramientas metodológicas utilizadas para delimitar las unidades de paisaje que fueron sujetas a valoración mostraron que en el manejo de la selva, los habitantes reconocen y nombran los espacios o (unidades) de su territorio que les proporcionan los recursos necesarios para vivir $\mathrm{y}$, por lo tanto, les atribuyen diferente nivel de importancia o valor. En la comparación e integración de las clasificaciones derivadas del conocimiento científico y la del conocimiento local, destacamos que el elemento de mayor peso para establecer los límites entre las unidades de paisaje fue el tipo de vegetación.

De acuerdo con Estrada-Lugo (2005), el ejido X-Hazil sur y anexos ha organizado su paisaje natural en diferentes espacios o territorios, según las características del ambiente y según el tipo de actividad que se puede realizar en el espacio. En otras palabras, la gente local puede reconocer, en el paisaje del ejido, los espacios idóneos para las diferentes actividades que realizan. Las comunidades practican un aprovechamiento diferencial de la selva, tienen una porción del paisaje para el aprovechamiento forestal, zonas o áreas para el uso agropecuario y zonas como la sabana inundable, así como los cenotes o las aguadas para otras actividades (Toledo et al., 2008; Estrada-Lugo et al., 2005; Macario-Mendoza, 2003; García-Frapolli et al., 2008). Este uso diferencial también fue encontrado en los ejidos de Noh Cah y X-Maben. En este sentido, Faust, 2001; Porter-Bolland $e t$ al., 2008 y Dalle et al., 2006 resaltan la importancia de tomar en cuenta el conocimiento local, por ejemplo, las clasificaciones locales botánicas, de suelos, de espacios que permiten una mejor integración y entendimiento del manejo comunitario por los mayas sobre sus recursos naturales.

La delimitación de los seis espacios o unidades resultó de utilizar principalmente las coincidencias que existían tanto en los mapas etnográficos como en las clasificaciones de Santiago-Cruz (2000), MacarioMendoza (2003) y Dalle et al. (2006). En la literatura, la milpa ocupa un lugar en las clasificaciones por uso de suelo; sin embargo, para esta investigación se decidió no incluirla como una unidad para evitar sesgar los resultados, debido a que la milpa es la base de la alimentación en la zona maya y, por tanto, la principal actividad agrícola, lo que seguramente la pondría en el primer lugar de importancia, además de que sus ubicaciones no son fijas, es decir, se van rotando entre los acahuales o hubchés. Pero en nuestro caso, para incluir el potencial agrícola se incluyeron dos tipos de acahuales, los de cinco a 10 años y los de 25 años o más. 
En investigaciones similares, García-Frapolli et al. (2008) y Toledo et al. (2008) en sus estudios emplean el uso de unidades productivas de paisaje en comunidades mayas de Yucatán, con el objetivo de documentar los procesos de apropiación de la naturaleza en estas comunidades. Sus unidades fueron la milpa, los huertos familiares, las selvas secundarias, los sistemas acuáticos y la selva madura, las cuales fueron agrupadas de acuerdo con los usos; las dos primeras corresponderían a un medioambiente transformado (МАT), las selvas secundarias y los cuerpos de agua a un medioambiente usado (MAU) y la selva madura a un medio ambiente conservado (MAC).

Aunque reconocemos que esta clasificación también es aplicable para nuestra zona de estudio, debido a que el manejo de recursos naturales presenta muchas similitudes con la estudiada por García-Frapolli, et al. (2008) y Toledo et al. (2008), la presente investigación dio prioridad a la delimitación local, es decir, a la establecida por los informantes clave de cada comunidad, debido a que las unidades de estudio a valorar debían resultar ser reconocidas ante los habitantes de las comunidades.

\subsection{Escala de valoración y valores de uso y no uso para las unidades de paisaje}

De acuerdo con los resultados obtenidos del ejercicio de valoración de estas unidades, para los hombres de las comunidades estudiadas, el monte alto o selva madura fue la unidad más valorada porque es el lugar donde se encuentran los recursos necesarios para su subsistencia, esto a pesar de que actualmente ya no pueden realizar allí la milpa. El aprovechamiento forestal tradicional de las selvas por parte de los mayas de Quintana Roo se realizó desde el establecimiento de los primeros habitantes en la región, ya que tuvieron que cubrir la necesidad de viviendas y alimentación (MacarioMendoza y Sánchez-Pérez, 2011). Mientras que el aprovechamiento forestal comercial en el que incursionan estás comunidades y principalmente $\mathrm{X}$-Hazil sur data de mediados de los ańos cincuenta durante las concesiones forestales, tiempo en que la empresa Maderas Industrializadas de Quintana Roo (MIQRoo) fue la principal beneficiada.

En 1983 se implementó el Plan Piloto Forestal de Quintana Roo, basado en la participación local, es decir, compartir entre todos los participantes el poder en la toma de decisiones. Cuando comenzó su implementación, a partir de una asamblea ejidal, se estableció una parte de la superficie de dotación ejidal como Área Forestal Permanente (AFP), y así, ellos comenzaron a involucrarse en la administración y conservación de sus selvas. Inicialmente, la venta era de madera en pie, pero pronto comenzaron a realizar la corta y venta ellos mismos. Este programa ha au- 
mentado los ingresos económicos de los campesinos y estimulado la organización y participación de los ejidos en el manejo y explotación de la selva (Murphy, 1992).

Fue en 1994, cuando el gobierno federal a través del Procampo restringió el establecimiento de milpas en el monte alto, pero otorgó apoyos monetarios para las actividades agrícolas; sin embargo, los participantes de las tres comunidades reconocen que lo anterior derivó en un incremento en el uso de agroquímicos en los acahuales jóvenes principalmente (impacto que tendrían que evaluar las investigaciones en conservación del manto freático en el estado). Los agroquímicos facilitan las labores agrícolas y responden a que los sitios cercanos a los poblados o a las carreteras donde se puede hacer milpa están compuestos principalmente de acahuales que no completan el periodo de descanso que requiere el sistema de roza-tumba y quema para que el suelo recupere su fertilidad. Los participantes en los talleres mencionaron que si les permitieran hacer milpa en el monte alto, sus cultivos serían mejores y no requerirían de herbicidas para mantenerlos limpios ni fertilizantes porque en el monte alto, la tierra es buena.

Este resultado, relacionado con la importancia de tener una milpa exitosa para los mayas de Quintana Roo, también ha estado reflejado en previos ejercicios de valoración no monetaria en la zona. En particular, corresponde con lo obtenido por Arce-Ibarra (2007), quien reporta que para estas comunidades los suelos para la milpa fueron los recursos más importantes durante su ejercicio de valoración. La anterior investigación y diversos autores que estudian esta zona mencionan que la relevancia de este policultivo es múltiple, lo cual se refleja en la alimentación local y, en general, en la cultura maya.

Por ejemplo, la importancia cultural de la milpa se aprecia en las ceremonias religiosas relacionadas con las prácticas agrícolas que son desarrolladas de manera comunitaria mientras que el valor social corresponde con aspectos de tenencia de la tierra, relaciones de reciprocidad y otras interacciones sociales (Bello-Baltazar, 2001; Estrada-Lugo, 2005; Toledo et al., 2008; García-Frapolli et al., 2008). Además, los hogares de estas comunidades suelen dedicar gran parte de sus horas laborables a las diferentes actividades que se desarrollan con la milpa (García-Frapolli et al., 2008) y, en general, entre los mayas se reporta la existencia del sistema de producción milpero, en el cual se realizan las actividades productivas de caza, pesca y recolección, tomando en cuenta el tiempo que deja disponible el cultivo de la milpa (Bello-Baltazar, 2001).

En este sentido, si retomamos los escenarios de daño usados para la evaluación del valor en las unidades seleccionadas, en caso de que el monte alto recibiera algún daño, se reconoce que su recuperación sería 
más larga, a diferencia de las otras unidades de paisaje, es decir, en términos económicos, esta elección de mayor importancia del monte por los grupos de varones mayas resalta diferentes tipos de valor de la selva madura, como son el de sustento-doméstico y el económico (crematístico), pero también el de opción y el de existencia (ver cuadro 11).

El aprovechamiento forestal de maderas preciosas y blandas es otra de las actividades relevantes llevadas a cabo en el monte alto y que para los ejidos representa una fuente de ingresos económicos muy importante. El manejo forestal que se lleva a cabo en la zona es de acuerdo a las especificaciones de los planes de manejo de cada ejido. Como se mencionó anteriormente, actualmente sólo el ejido X-Hazil sur y anexos tiene permiso para esta extracción, mientras que los otros ejidos estudiados sólo pueden extraer productos no maderables.

Dalle et al. (2006) resaltan que en el ejido de X-Maben las restricciones para llevar a cabo su milpa en el monte alto movieron la agricultura hacia el monte bajo y hacia los acahuales jóvenes, a pesar de ser menos productivos para la milpa. En los talleres realizados en la comunidad Señor, mencionaron que esto también se relaciona con la cercanía de estos sitios a los poblados, ya que se facilita el transporte de los productos al hogar.

Como se observó en la sección de resultados, los acahuales de 20 años ocuparon el segundo lugar para los hombres en las tres comunidades, debido al tiempo que llevan de recuperación lo que se relaciona con un suelo más productivo. Para esta unidad, un elemento conspicuo de este paisaje mencionado reiteradamente en los talleres fue la palizada, que se refiere a un conjunto de maderas largas y delgadas de tamaños similares utilizadas para la construcción de las casas tradicionales, y también, como lo menciona Arce-Ibarra (2007), son útiles durante las fiestas tradicionales para la construcción de los corrales y, finalmente, son una fuente de ingreso por la construcción de palapas para la industria de turismo regional. En este sentido, lo anterior es reflejo de valor cultural inmerso en esta unidad de paisaje. De forma similar a lo encontrado en nuestro estudio, Arce-Ibarra (2007) expone que en su ejercicio de valoración, la palizada ocupó el segundo lugar de importancia entre los recursos útiles en las comunidades de X-Maben.

Con respecto a las diferencias de género entre los grupos comunitarios participantes, encontramos que para las mujeres las comunidades Noh Cah y Señor, los acahuales de 20 años o más ocuparon el primer lugar de importancia. En este sentido, tanto mujeres como hombres argumentaron que esta diferencia se debe a que ellas casi no trabajan en el campo ni han recorrido todo el ejido, por lo tanto, su elección se debe principalmente a que es el lugar que conocen cuando acompañan a sus esposos porque es donde se permite trabajar la milpa, y allí se realiza también la extracción 
de diferentes productos maderables. García-Frapolli et al. (2008) mencionan que en las comunidades de mayas yucatecos que trabajaron, la principal actividad de las mujeres se centra en la administración del hogar, lo que implica labores en el huerto, en la educación y alimentación de los hijos, así como apoyar en labores de la milpa. Estas actividades también se reflejan en las comunidades bajo estudio y pueden sustentar las diferencias entre escalas.

Otra diferencia encontrada entre mujeres y hombres de las comunidades estudiadas fue la valoración de los cuerpos de agua, los cuales tuvieron mayor importancia para las primeras. Con relación a esto, en las tres comunidades se mencionó durante las entrevistas y en los talleres que las mujeres se reúnen en grupo para visitar las lagunas; en las observaciones realizadas en campo también se presenciaron estas actividades.

Con respecto a los cuerpos de agua del centro de Quintana Roo, es importante destacar que su uso principal es la pesca de autoconsumo, actividad que depende principalmente del tamaño y profundidad de los cuerpos de agua, ya que estas características permiten la existencia de fauna acuática temporal o permanente (Ramírez-Barajas et al., 2001). Así, la pesca forma parte de la diversidad de actividades que los habitantes de estas comunidades realizan para cubrir sus necesidades de autoconsumo, enriquecer su dieta, obtener productos de alto valor de uso que ocasionalmente derivan en ingresos monetarios cuando se venden dentro de la comunidad (Estrada-Lugo y Arce-Ibarra, 2011). De manera particular, en la comunidad Señor y Noh Cah se comentó que la actividad es sólo de autoconsumo, es decir, no hay comercio dentro o fuera de la comunidad, mientras que en X-Hazil sur, sí hay venta (Rojas-García, 1999).

En los valores históricos y culturales inmersos en el uso de los cuerpos de agua se ha reportado la existencia de prácticas ceremoniales al iniciar la jornada de pesca éstas consisten en el ofrecimiento de comidas y bebidas especiales para la ocasión, más en el sentido de ofrendar a las deidades del monte y de los cenotes, así como al santo para solicitar una jornada segura y productiva (Estrada-Lugo y Arce-Ibarra, 2011). Durante las entrevistas realizadas se evidenció el respeto hacia estas unidades a través de reglas locales, por ejemplo, en la comunidad Noh Cah y Señor se sugiere guardar silencio, de lo contrario uno se encontraría en riesgo de contraer alguna enfermedad como castigo por parte de los dueños de los cuerpos de agua.

El monte bajo y los acahuales de cinco a 10 años tuvieron una importancia intermedia para los entrevistados. Los hombres mencionaron que en los acahuales de cinco a 10 años las arvenses crecen más rápido, lo que incrementa las labores de limpia, pero que usando herbicidas ese problema queda resuelto. Cabe destacar que reconocen que los herbici- 
das dañan también sus cultivos y la vegetación de los alrededores, pero mencionan que prefieren eso a tener que limpiar con más frecuencia la milpa. En este sentido, García-Romero et al. (2003), mencionan que los acahuales jóvenes tienen bajos niveles de productividad y mayor sensibilidad al impacto de la lluvia y el viento, lo que limita su uso óptimo con fines agrícolas.

Comparado con las otras cinco unidades de paisaje seleccionadas, la sabana fue el espacio menos valorado para todos los grupos de interés. Las razones para lo anterior se relacionan con que la única actividad que se puede realizar ahí es la cacería. Además se mencionó que los suelos no son útiles para la siembra. También se mencionó que en Señor y X-Hazil sur se han realizado ensayos de siembra de arroz pero que debido a la dificultad de conseguir la semilla no se ha continuado esta actividad. Hace más de 40 años en esta unidad se realizaba la cacería de los cocodrilos para la venta de la piel y consumo de su carne (Zamudio et al., 2011).

Los resultados de valoración obtenidos de los expertos académicos en la zona maya sólo difieren significativamente con los obtenidos para el grupo de hombres de la comunidad Noh Cah, es decir, la experiencia de las investigaciones en recursos naturales y en ciencias y humanidades que han tenido en la zona los participantes en el grupo de expertos, muestra que ellos entienden o perciben una valoración de unidades de paisaje muy similar a la de las comunidades mayas de Quintana Roo. La relevancia de lo anterior radica en que, si no todas, muchas de las investigaciones de este grupo pueden o deben ser incorporadas al desarrollo regional y a las políticas públicas ambientales, aspecto en lo que poco se ha avanzado en Quintana Roo (Faust, 2001; Bello-Baltazar, 2001).

En general, puede decirse que la valoración de las unidades de paisaje sirve primero para conocer cómo perciben las comunidades mayas a su ejido, es decir, con estos resultados se conoce la opinión de diferentes personas de la comunidad (hombres y mujeres) sobre la importancia de los espacios de su ejido. Los resultados y la literatura revisada muestran que la estrategia maya gira alrededor de varias prácticas productivas, cuya intensificación depende no sólo de factores como la demografía sino cada vez más, respecto a la gama de oportunidades mercantiles y al acceso de los nuevos mercados, así como a la cosmovisión que marca las actividades en una lógica sagrada de respeto, en donde determinadas prácticas culturales tienen un papel preponderante (Toledo et al., 2008).

Por otro lado, la valoración de estas unidades de paisaje, las cuales no tienen un mercado establecido da cabida a establecer incentivos o pagos (futuros) consistentes con la importancia que le da la comunidad, por ejemplo, para establecer pagos por daños, naturales o inducidos, a los recursos naturales locales. Con los resultados de nuestro estudio, y ante 
una evaluación futura de daños provocados a las unidades evaluadas, las comunidades mayas pedirían una mayor compensación, por ejemplo, por el monte alto que por la sabana (Rutherford et al., 1998). Nuestros resultados también son útiles para priorizar en los esfuerzos de conservación de las selvas de Quintana Roo con políticas que estén acordes con la lógica de uso múltiple de los recursos locales. Finalmente, Chuenpagdee et al. (2002) destacan que el conocimiento de la valoración de la comunidad hacia los recursos naturales, previos al desarrollo de planes o programas basados en éstos, es importante para tener éxito en su implementación y desarrollo, ya que facilita el consenso entre los diferentes actores involucrados en estos procesos. Por lo tanto, la valoración de las unidades de paisaje será útil para quienes quieran llevar a cabo nuevos proyectos productivos en la zona, o bien, para planificar el uso de estos espacios desde enfoques más integrales, tomando en cuenta los usos o actividades que en este caso sean coincidentes con los resultados de la valoración.

\subsection{Servicios ecológicos de las unidades de paisaje}

Termorshuizen y Opdam (2009) establecen que los servicios ecológicos y de bienestar como la producción de alimento, regulación del clima, educación, así como la reproducción de prácticas culturales, tienden un puente o conexión entre los valores locales y el uso sostenible del paisaje. En este sentido, se resalta la importancia de que las comunidades reconozcan los beneficios o los servicios ecológicos y de bienestar que las unidades de paisaje les proporcionan. A lo largo de esta investigación, las personas mencionaron algunos servicios ecológicos y de bienestar, como los relacionados con la provisión, que de acuerdo con la Evaluación de los Ecosistemas del Milenio o del Millennium Ecosystem Assessment (MEA, 2005), incluyen todo el material y las salidas energéticas de los ecosistemas, los cuales son tangibles y pueden ser intercambiados, vendidos o usados directamente por la gente.

Así, estos servicios se relacionan con el aprovechamiento integral de los recursos naturales por parte de los mayas macehuales, principalmente los que se derivan del conocimiento local sobre el uso histórico de sus recursos naturales generado a través de procesos de ensayo y error. Este conocimiento es transmitido de generación en generación, donde se involucran la agricultura tradicional (milpa), la recolección de una amplia variedad de productos maderables y no maderables, la cacería de fauna silvestre y la pesca (Rojas, 1999; Bello-Baltazar, 2001; Macario-Mendoza, 2003; Estrada-Lugo, 2005; Arce-Ibarra y Charles 2008; García-Frapolli et al., 2008; Toledo et al., 2008). 
De tal manera que el conocimiento local o etnoecológico se ha ido fortaleciendo también gracias a la investigación participativa de varios científicos que trabajan en la zona maya, por ejemplo, en Noh Cah mencionaron la importancia de cuidar los árboles de la selva porque producen oxígeno, pero también porque retienen el suelo que de no hacerlo se van a su laguna provocando su azolvamiento. Este conocimiento lo atribuyen principalmente a la asistencia técnica de científicos que laboran en la zona maya, en particular, por el trabajo realizado por Elías-Gutiérrez et al. (2004). Estos autores llevaron a cabo la evaluación ambiental de la Laguna Tortugas de Noh Cah y presentaron los resultados en un taller comunitario, usando diapositivas pero también un microscopio para presentar los microorganismos que son el alimento de los peces de esa laguna.

En la última década en la zona maya se han implementado nuevos proyectos o programas de carácter público como el pago por servicios ambientales por regulación hídrica o captura de carbono del CompactPNUD (s.p.i.). Otro ejemplo de estos programas es el del ejido Noh Cah que incluye el pago por servicios ambientales de la Conafor, y que cuenta con un área de reserva hidrológica, y en las entrevistas abiertas se resaltó la importancia de conservar la selva con este fin. En nuestro trabajo de campo se constató que en el ejido X-Hazil sur y anexos también se implementó este programa, mientras que en X-Maben aún no se implementa.

En general, los programas mencionados también contribuyen a complementar el conocimiento sobre los servicios ecológicos y de bienestar en las comunidades. Específicamente el servicio de captura de carbono, desde el punto de vista científico, es de los más importantes en los ecosistemas tropicales. Al respecto de esto, Martínez-Bravo y Masera (2008) reportan que la sabana -la unidad menos valorada en nuestro estudiotiene una capacidad de almacenamiento de carbono aproximado de 149 t/ha, mientras que los suelos de la selva madura presentan un aproximado de 57 t/ha de $\mathrm{C}$, al igual que los acahuales maduros. Además de que, en términos ecológicos, la sabana se reconoce como una zona de transición entre los ecosistemas acuáticos y terrestres, por lo que diversas especies terrestres y acuáticas ocupan estos sitios como refugio relacionado con el valor ecológico reconocido por los habitantes de las comunidades, a pesar de que para todos los grupos de interés esta fue la unidad de menor importancia.

Con respecto a los servicios culturales, las comunidades resaltaron la importancia del monte alto en donde aún se llevan a cabo ceremonias y rezos que se relacionan con un uso respetuoso de los recursos de la selva. Por otro lado, se sabe que en los ejidos de X-Hazil sur y anexos y XMaben se cuenta con áreas destinadas para el turismo de naturaleza. En 
particular en X-Maben, esta área es una reserva de 100 ha conocida con el nombre de Laguna Azul, ubicada a ocho kilómetros del poblado Señor. En esta zona se han encontrado vestigios de los mayas ancestrales que son un atractivo para el turismo. El grupo local X'yaat, con oficinas ubicadas en la comunidad Señor, se dedica al llamado ecoturismo o turismo alternativo, ofrece recorridos en la laguna en canoa o kayac, así como la observación de aves y campamentos. También dan recorridos culturales, es decir, llevan a los turistas a la comunidad para que conozcan sus formas de vida, ofreciéndoles alimentos típicos y hablándoles de algunas prácticas sociales comunes en la zona maya.

\section{Conclusiones}

El mosaico actual que constituyen los paisajes es producto innegable de la historia, incluye huellas de los efectos culturales que surgen a diferentes escalas espaciales y temporales (Nassauer, 1997). En nuestro estudio, los componentes espaciales están condicionados por una combinación de factores biofísicos y socioeconómicos que confluyen en el territorio, algunos efectos se identifican claramente en paisajes que han sido modificados y formados por razones productivas como los paisajes rurales (Lafortezza et al., 2008). En este sentido, la delimitación de unidades de paisaje en esta investigación permite relacionar el mosaico de vegetación en diferentes estados de sucesión con el manejo local diversificado de los recursos naturales disponibles, donde se realizan actividades con orígenes prehispánicos como la milpa hasta actividades impulsadas por agentes externos como es el caso de la extracción de maderas preciosas para el mercado nacional e internacional. En todos los casos, los resultados de la valoración no monetaria estuvieron en correspondencia directa con el potencial que tienen las unidades de paisaje para satisfacer las necesidades de subsistencia de las comunidades mayas estudiadas.

El monte alto y el acahual de 20 años o más representaron las unidades de valor más alto para los grupos de interés, porque cuentan con los elementos necesarios para el sustento de los mayas macehuales. Son espacios donde se reconocen valores de sustento-doméstico, económicos, de opción y existencia. Todos los valores reconocidos por los mayas macehuales se relacionan con un uso respetuoso de la naturaleza. Las ceremonias y los rezos a los seres que cuidan el monte son todavía parte de una cultura que ha prevalecido por más de 3,000 años en la península de Yucatán.

Los cuerpos de agua, el monte bajo y el acahual de cinco a 10 años formaron el grupo de valor intermedio. El lugar que ocupaban las unidades en el orden de cada escala dependía de determinadas actividades que diferenciaban a cada grupo de interés; sin embargo, las variaciones encon- 
tradas entre los grupos fueron muy sutiles. La diferencia más sobresaliente entre la valoración que dan los hombres y las mujeres a las unidades se puede atribuir, principalmente, a los diferentes roles sociales que desarrollan en la comunidad, es decir, los hombres conocen más el campo y los terrenos de todo el ejido porque allí laboran cotidianamente, mientras que las mujeres se identifican más con labores hogareñas, pero conocen el campo ya que acompañan a sus esposos a la milpa. Finalmente, la sabana fue la unidad menos importante para las comunidades estudiadas y es donde se realizan las menores actividades de aprovechamiento de recursos naturales.

En general, las comunidades mayas relacionaron la importancia de las unidades de paisaje con los servicios ecológicos y de bienestar que éstas les proporcionan, por ejemplo, la generación de oxígeno, hábitat para los animales, producción de alimento, así como con la importancia en la reproducción de sus prácticas culturales que soportan su identidad como mayas macehuales.

La utilidad de los resultados de la presente investigación es múltiple, por ejemplo, en el caso de manejo, conservación de recursos naturales y de desarrollo regional, el uso de las preferencias y de los valores del paisaje de la población local como instrumento de conexión a los sitios de un territorio, ayuda tanto a planificar el uso sostenible del territorio como a establecer escalas previas de compensación por daños potenciales futuros a recursos locales.

\section{Agradecimientos}

Al Fondo Institucional de Fomento Regional para el Desarrollo Científico, Tecnológico y de Innovación (Fordecyt) del Consejo Nacional de Ciencia y Tecnología a través del Convenio 116306: Innovación Socioambiental para el Desarrollo en Áreas de Alta Pobreza y Biodiversidad de la Frontera Sur de México (Redisa).

A las autoridades y a la población de los ejidos Noh Cah, X-Hazil Sur y Anexos y X-Maben, gracias por su hospitalidad y apoyo. También agradecemos al grupo de académicos que labora en la zona maya por su disposición en las entrevistas.

\section{Bibliografía}

Araújo-Santana, Maria Raimunda, Manuel Roberto Parra-Vázquez, Ernesto Benito Salvatierra-Izaba, Ana Minerva Arce-Ibarra, Florencia Montagnini (2013), "Políticas turísticas, actores sociales y ecoturismo en la península de Yucatán”, Economía, Sociedad 
y Territorio, XIII (43), El Colegio Mexiquense, A.C., Zinacantepec, pp. 641-674.

Arce-Ibarra, Ana Minerva (2007), "Livelihoods, aquatic resources and Non-monetary values of local natural resources in Mexico's Lowland Maya area”, tesis de doctorado, Dalhousie University, Halifax.

Arce-Ibarra, Ana Minerva y Anthony Charles (2008), "Inland fisheries of the Mayan Zone in Quintana Roo, Mexico: using a combined approach to fishery assessment for data-sparse fisheries", Fisheries Research, 91, Elsevier, Amsterdam, pp. 151-159.

Barbier, Edward (1994), "Valuing environmental functions: tropical wetlands", Land Economics, 70 (2), Universidad de Wisconsin, Madison, pp. 155-173.

Bello-Baltazar, Eduardo (2001), "Milpa y madera, la organización para la producción entre mayas de Quintana Roo", tesis de doctorado, Universidad Iberoamericana, México.

Berkes, Fikret (1999), Sacred ecology: traditional ecological knowledge and resource management, Taylor \& Francis, Philadelphia.

Bernard, Harvey Russell (2006), Research methods in anthropology: qualitative and quantitative approaches, Altamira Press, Rowman \& Littlefield publishers, Lanham.

Brown, Gregory (2006), "Mapping landscape values and development preferences: a method for tourism and residential development planning", International Journal of Tourism Research, 8, Wiley, Oxford, pp. 101-113.

Brown, Gregory y Christopher Raymond (2007), “The relationship between place attachment and landscape values: toward mapping place attachment", Applied Geography, 27, Indiana State University, Terre Haute, pp. 89-111.

Buxó, Ramón (2006), "Paisajes culturales y reconstrucción histórica de la vegetación”, Ecosistemas, 15 (1), Asociación Espańola de Ecología Terrestre, Madrid, pp. 1-6. 
Chuenpagdee, Ratana (1998), "Damage schedules and scales of importance of losses: judgments of coastal resource users in Thailand", <http://203.116.43.77/publications/research1/ACFC9.html>, 3 de marzo de 2009.

Chuenpagdee, Ratana, Jack L. Knetsch y Thomas C. Brown (2002), "Environmental damage schedules: community judgments of importance and assessments of losses", Land Economics, 77 (1), University of Wisconsin, Madison, pp. 1-11.

Dalle, Sarah Paule, Sylvie de Blois, Javier Caballero y Timothy Johns (2006), "Integrating analyses of local land-use regulations, cultural perceptions and land-use/land cover for assessing the success of community-based conservation", Forest Ecology and Management, 222, Elsevier, Amsterdam, pp. 370-383.

Dunn-Rankin, Peter, Gerarld A. Knezek, Susan Wallace y Shuqiang Zhang (2004), Scaling methods, Lawrence Erlbaum Associates, New Jersey.

Elías-Gutiérrez, Manuel, Ana Minerva Arce-Ibarra, Aristeo HernándezSánchez (2004), "Primera evaluación ambiental de la Laguna de Noh Cah", documento de trabajo, Centro de Información y Documentación unidad regional Quintana Roo, Quintana Roo.

Elías-Gutiérrez, Manuel, Adrián Cervantes-Martínez, Martha GutiérrezAguirre y Ana Minerva Arce-Ibarra (2007), "Los cenotes y lagunas del centro y sur de la península de Yucatán”, en Guadalupe de la Lanza Espino (comp.), Las aguas interiores de México: conceptos y casos, AGT Editor, México, pp. 423-446.

Estrada-Lugo, Erin Ingrid Jane (2005), "Grupo doméstico y usos del parentesco entre los mayas macehuales del centro de Quintana Roo: el caso del ejido X-Hazil y anexos", tesis de doctorado, Universidad Iberoamericana, México.

Estrada-Lugo, Erin Ingrid Jane y Ana Minerva Arce-Ibarra, (2011), "La pesca entre los mayas macehuales del centro de Quintana Roo", en Erin Ingrid Jane Estrada-Lugo y Eduardo Bello-Baltazar (comps.), Cultivar el territorio maya. Conocimiento y organización social en el uso de la selva, Universidad Iberoamericana, México, pp. 189-212. 
Faust, Betty Berenice (2001), "Maya environmental successes and failures in the Yucatan peninsula", Environmental Science and Policy, 4, Geneva University, Geneva, pp. 153-169.

Fuente-Carrasco, Mario E. (2008), "La economía ecológica: ¿un paradigma para abordar la sustentabilidad?", Argumentos. Estudios Criticos de la Sociedad, 56, Universidad Autónoma Metropolitana unidad Xochimilco, México, pp. 75-99.

García-Romero, Arturo, Karla Ivette Mendoza-Robles y Leopoldo Galicia-Sarmiento (2003), "Valoración del paisaje de la Selva Baja Caducifolia en la cuenca baja del río Papagayo (Guerrero), México (Parte B)", Investigaciones Geográficas, 56, Universidad Nacional Autónoma de México, México, pp. 77-100.

García-Frapolli, Eduardo, Víctor Manuel Toledo y Joan Martínez-Alier (2008), "Apropiación de la naturaleza por una comunidad maya yucateca: un análisis económico ecológico", Revista Iberoamericana de Economía Ecológica, 7, Red Iberoamericana de Economía Ecológica, Barcelona, pp. 27-42.

Gómez-Baggethum, Erik y Rudolf de Groot (2007), "Capital natural y funciones de los ecosistemas: explorando las bases ecológicas de la economía”, Ecosistemas, 16 (003), Asociación Española de Ecología Terrestre, Alicante, pp. 4-14.

Granato, Leonardo, Nahuel Oddone y Adolfo Carballo-Penela (2009), "Valoración económica del medio ambiente. Las propuestas de la economía ecológica y la economía ambiental”, Revista OIDLES, 3 (7), Grupo Eumed.net, Universidad de Málaga, Málaga, pp. 1-11.

Grêt-Regamey, Adrianne, Ariane Walz y Peter Bebi (2008), "Valuing ecosystem services for sustainable landscape planning in Alpine regions", Mountain Research and Development, 28 (2), International Mountain Society, Bern, pp. 156-165.

INEGI (Instituto Nacional de Estadística, Geografía e Informática) (2006), II Conteo de Población y Vivienda 2005. Principales resultados por localidad, 2005, INEGI, México, <http://www.inegi.org.mx>, 6 de febrero de 2010. 
Jiménez-Osornio, Juan José, Arturo Caballero, Delfín Quezada y Eduardo Bello-Baltazar (2003), "Estrategias tradicionales de apropiación de los recursos naturales", en Patricia Colunga García-Marín y Alfonso Larque-Saavedra (eds.), Naturaleza y sociedad en el área maya. Pasado, presente y futuro, Academia Mexicana de Ciencias, Mérida, pp. 189-200.

Kramer, Randall A., Evan Mercer y Sharma Narendra (1996), "Valuing tropical rainforest protection using the contingent valuation method", en Wiktor Adamowicz, Peter Boxall, Martin Luckert, William Philipps y William White, Forestry, Economics and the Environment, СAB International, Wallingford, pp. 181-194.

Lafortezza, Raffaele, Robert C. Corry, Giovanni Sanesi y Robert D. Brown (2008), "Cultural determinants of spatial heterogeneity in forest landscapes", en Raffaele Lafortezza, Giovanni Sanesi, Jiquan Chen y Thomas Crow (eds.), Patterns and processes in forest landscapes. Multiple use and sustainable management, Springer, Dordrecht, pp. 17-32.

Lazos, Elena y Luisa Paré (2000), Miradas indígenas sobre una naturaleza entristecida: percepciones del deterioro ambiental entre nahuas del sur de Veracruz, Plaza y Valdés-Universidad Nacional Autónoma de México, México.

López-Barajas, René y Jorge Cervantes-Borja (2002), "Unidades del paisaje para el desarrollo sustentable y manejo de los recursos naturales", Notas. Revista de Información y Análisis, (20), Instituto Nacional de Estadística Geografía e Informática, México, pp. 43-49.

López-Hernández, José Rudier, Eduardo Bello-Baltazar, Erin Ingrid Jane Estrada-Lugo, Marie Claude Brunel-Manse, César Adrián RamírezMiranda (2012), "Instituciones locales y procesos organizativos: el caso de la Reserva de la Biósfera Sian Ka’an”, Estudios Sociales, 41, Coordinación de Desarrollo Regional del Centro de Investigación en Alimentación y Desarrollo, Asociación Civil, Hermosillo, pp. 66-93.

Macario-Mendoza, Pedro Antonio (2003), "Efecto del cambio de uso de suelo sobre la selva y estrategias para el manejo sustentable de la vegetación secundaria en Quintana Roo", tesis de doctorado, Universidad Autónoma de Yucatán, Mérida. 
Macario-Mendoza, Pedro Antonio y Luis Candelario Sánchez-Pérez (2011), "Recolección de plantas: uso forestal tradicional y extracción forestal comercial en una comunidad maya del centro de Quintana Roo", en Erin Ingrid Jane Estrada-Lugo, Eduardo Bello-Baltazar (comps.), Cultivar el territorio maya. Conocimiento y organización social en el uso de la selva, Universidad Iberoamericana, México, pp. 203-236.

Martínez-Bravo, René y Omar Masera (2008), "La captura de carbono como servicio ecosistémico del Parque Ecológico Jaguaroundi: una estrategia para la conservación y manejo de los recursos forestales", en Yolanda Nava e Irma Rosas (coords.), El Parque Ecológico Jaguaroundi. Conservación de la selva tropical veracruzana en la zona industrializada, Secretaría de Medio Ambiente y Recursos Naturales-Instituto Nacional de Ecología-Petróleos Mexicanos Petroquímica-Universidad Nacional Autónoma de México, México, pp. 101-114.

MEA (Millennium Ecosystem Assessment) (2005), Millennium Ecosystem Assessment Report, Island Press, Washington, DC.

Mejía-Ávila, Doris (2007), “Delimitación de las unidades de paisaje del parque Nacional Natural Paramillo", Revista Electrónica Hominis, vol. 1, Universidad de Córdoba, Córdoba, pp. 8-17.

Miranda, Faustino y Efraín Hernández-Xolocotzi (1963), "Los tipos de vegetación de México y su clasificación”, Boletín de la Sociedad de Botánica de México, (28), Sociedad Botánica de México, México, pp. 29-179.

Murphy, Julia E. (1992), “Aprovechamiento forestal y la agricultura de milpa en el ejido X-Maben, zona maya de Quintana Roo, México", en Laura K. Snook, Amanda Barrera de Jorgenson (eds.), Memorias del taller Madera, chicle, caza y milpa. Contribuciones al manejo integral de las selvas de Quintana Roo, México, ProaftINIFAP-USAID, WWF us, México, pp. 3-18.

Nahuelhual, Laura, Pablo Donoso, Antonio Lara, Daisy Nuñez, Carlos Oyarzu y Eduardo Neira (2006), "Valuing ecosystem services of chilean temperate rainforests", Environment, Development and Sustainability, 9 (4), Springer, Dordrecht, pp. 481-499. 
Nassauer, Joan Iverson (1997), Placing nature. Culture and landscape ecology, Island Press, Washington, DC.

Patel, Aviva, David Joseph Rappaport, Loren Vanderlienden y John Eyles (1999), "Forests and societal values: comparing scientific and public perception of forest health", The Environmentalist, 19 (3), Kluwer Academic Publishers, Boston, pp. 239-249.

Pool, Novelo Luciano, Noe Samuel León-Martínez, Pedro Antonio Macario-Mendoza, María Angélica Navarro-Martínez y Ramón Mariaca-Méndez (2003), "Ambiente y recursos naturales”, en Guillermo Montoya, Eduardo Bello, Manuel Parra y Ramón Mariaca, La frontera olvidada entre Chiapas y Quintana Roo, Consejo Estatal para la Cultura y las Artes de Chiapas-El Colegio de la Frontera Sur, Tuxtla Gutiérrez, pp. 69-92.

Porter-Bolland, Luciana, Edward A. Ellis and Henry L. Gholz. (2007), "Land use dynamics and landscape history in La Montaña, Campeche, Mexico", Landscape and Urban Planning, (82), Elsevier, Amsterdam, pp. 198-207.

Porter-Bolland, Luciana, María Consuelo Sánchez-González y Edward Allan Ellis (2008), "La conformación del paisaje y el aprovechamiento de los recursos naturales por las comunidades mayas de La Montaña, Hopelchén, Campeche”, Investigaciones geográficas, Boletín del Instituto de Geografía, 66, Universidad Nacional Autónoma de México, México, pp.65-80.

Ramírez-Barajas, Pablo, Nuria Torrescano-Valle, Alejandro Tecpa-Jiménez, Juan Vázquez-Rodríguez (2001), "Importancia y uso del entorno natural en una comunidad maya (Petcacab, Quintana Roo, México)", Tip. Revista Especializada en Ciencias Quimico-Biológicas, 4, Universidad Nacional Autónoma de México, México, pp. 61-71.

Ran (Registro Agrario Nacional) (1995), Plano General del Ejido Noh Cah, Quintana Roo, Programa de Certificación de Derechos Ejidales y Titulación de Solares Urbanos, Ran, México.

RAN (Registro Agrario Nacional) (1998), Plano general del ejido X-Hazil, Quintana Roo, Programa de Certificación de Derechos Ejidales y Titulación de Solares Urbanos, Ran México. 
Rojas-García, Javier (1999), "Los recursos pesqueros del ejido X-Hazil sur y anexos, Quintana Roo, México: un estudio etnobiológico”, tesis de maestría, El Colegio de la Frontera Sur, Chetumal.

Rutherford, Murray B., Jack L. Knetsch y Thomas C. Brown (1998), "Assessing environmental losses: judgments of importance and damage schedules", Harvard Environmental Law Review, 22, Harvard Law School Student Journals Office, Cambridge, pp. 51-101.

Santos-Fita, Dídac, Eduardo J. Naranjo Piñera y José Luis Rangel-Salazar (2012), "Wildlife uses and hunting patterns in rural communities of the Yucatan peninsula, Mexico", Journal of Ethnobiology and Ethomedicine, 8 (38), BioMed Central, London, pp. 1-18.

Santiago-Cruz, Blas (2000), "Caracterización y uso actual del suelo en el ejido X-Hazil y anexos, Quintana Roo, México”, tesis de licenciatura, El Colegio de la Frontera Sur, Chetumal.

Termorshuizen, Jolande.W. y Paul Opdam (2009), "Landscape services as a bridge between landscape and sustainable development", Lanscape Ecology, 24, Springer, Dordrecht, pp. 1037-1052.

Toledo, Víctor Manuel (2001), "Biodiversity and indigenous peoples”, en S. Levin Simon (ed.), Encyclopedia of biodiversity, Academic Press, London, pp. 1181-1197.

Toledo, Víctor Manuel, Narciso Barrera-Bassols, Eduardo García-Frapolli y Pablo Alarcón-Chaires (2008), "Uso múltiple y biodiversidad entre los mayas yucatécos (México)", Interciencia, 33 (5), Asociación Interciencia, Caracas, pp. 345-352.

Vatn, Arild y Daniel W. Bromley (1994), “Choices without prices without apologies", Journal of Environmental Economics and Management, 26, Elsevier, San Diego, pp. 129-148.

Vera-Méndez, Paola Guadalupe (2008), “Análisis de los valores ambientales del Parque Nacional y Zona de Monumentos Arqueológicos Palenque, Chiapas", tesis de maestría, El Colegio de la Frontera Sur, San Cristóbal de las Casas.

Zamudio, Fernando, Eduardo Bello-Baltazar y Erin Ingrid Jane EstradaLugo (2011), “Integrando conocimientos mayas y científicos 
sobre el lagarto (Crocodylus moreleti) en el ejido X-Hazil sur y anexos, Quintana Roo, México", en Erin Ingrid Jane EstradaLugo y Eduardo Bello-Baltazar (comps.), Cultivar el territorio maya. Conocimiento y organización social en el uso de la selva, Universidad Iberoamericana, México, pp. 161-188.

Recibido: 26 de mayo de 2011. Reenviado: 23 de septiembre de 2011. Reenviado: 28 de noviembre de 2011. Aceptado: 24 de febrero de 2012.

Karla Diana Infante-Ramírez. Es bióloga, egresada de la Universidad Autónoma Metropolitana unidad Xochimilco. Fue condecorada con la medalla al mérito académico. Realizó la maestría en ciencias en recursos naturales y desarrollo rural en El Colegio de la Frontera Sur unidad Chetumal. Es estudiante del doctorado en ecología y desarrollo sustentable en El Colegio de la Frontera Sur unidad Chetumal (2014-2017). Su línea de estudio actual está orientada a la gestión de ecosistemas y territorios.

Ana Minerva Arce-Ibarra. Es doctora en estudios interdisciplinarios por la Universidad de Dalhousie (Canadá), maestra en biología marina por el Cinvestav-Mérida y licenciada en biología pesquera por la Universidad Autónoma de Sinaloa. Es miembro del Comité de Evaluación de Proyectos Compact-PNud-península de Yucatán. Su línea de investigación se centra en el análisis de las pesquerías artesanales por medio de estudios interdisciplinarios. Entre sus publicaciones destacan, en coautoría: "Nonmanagement of natural resources: the case of inland fisheries in the mayan zone, Quintana Roo, Mexico", Human Ecology, 36, Kluwer Academic, New York, pp. 853-860 (2008); "Inland fisheries of the mayan zone in Quintana Roo: using a combined approach to fishery assessment for data-sparse fisheries", Fisheries Research, 91, Elsevier, Amsterdam, pp. 151159 (2008); "Linking social and natural sciences methods using Mind Maps: a case study of human-nature interactions in Mexico's Lowland Maya area", The International Journal of Interdisciplinary Social Sciences, 2, Common Ground, Melbourne, pp. 39-52 (2007).

Eduardo Bello-Baltazar. Es doctor en antropología social por la Universidad Iberoamericana, maestro en ciencias con especialidad en desarrollo rural por el Colegio de Posgraduados, e ingeniero agrónomo por la Universidad Autónoma Metropolitana. Es investigador nivel i en el Sistema Nacional de Investigadores, actualmente labora en El Colegio de la 
Frontera Sur unidad San Cristóbal de Las Casas donde coordina la Red de Espacios de Innovación Socioambiental (Redisa). Investiga las implicaciones sociales del manejo de los recursos naturales en temas como: organización social y territorio, comunidad y planes de manejo del bosque (ecoturismo, silvicultura, productos no convencionales), ritualidad y redes sociales, leyes y normas locales. Entre sus publicaciones recientes destacan, en coautoría: "Experiencias, aprendizajes y diferencias en la toma de decisiones de dos grupos campesinos mayas en la adopción de tecnologías agrícolas en Sahcabá, Hocabá, Yucatán México”, Estudios de Antropología Biológica, XIV (II), Universidad Nacional Autónoma de México, México, pp. 629-647 (2009); "Forestería comunitaria y desarrollo de instituciones locales: el caso de la comunidad agraria Teopisca", Economía, Sociedady Territorio, IX (30), El Colegio Mexiquense, A.c., Zinacantepec, pp. 349395 (2009); "Religión y espacio social: una micro-región maya de Quintana Roo", en Julio Robertos, Ever Canal y Manuel Buenrostro (eds.), Mayas modernos de Quintana Roo, Universidad de Quintana Roo-Centro de Estudios Interculturales-Plaza y Valdés, pp. 25-55 (2008). 